\title{
Efficacy of Chinese herbal medicine for stroke modifiable risk factors: a systematic review
}

Wenbo Peng ${ }^{1}$, Romy Lauche ${ }^{1}$, Caleb Ferguson², Jane Frawley ${ }^{1}$, Jon Adams ${ }^{1}$ and David Sibbritt ${ }^{1,3^{*}}$

\begin{abstract}
Background: The vast majority of stroke burden is attributable to its modifiable risk factors. This paper aimed to systematically summarise the evidence of Chinese herbal medicine (CHM) interventions on stroke modifiable risk factors for stroke prevention.

Methods: A literature search was conducted via the MEDLINE, CINAHL/EBSCO, SCOPUS, and Cochrane Database from 1996 to 2016. Randomised controlled trials or cross-over studies were included. Risk of bias was assessed according to the Cochrane Risk of Bias tool.

Results: A total of 46 trials (6895 participants) were identified regarding the use of CHM interventions in the management of stroke risk factors, including 12 trials for hypertension, 10 trials for diabetes, eight trials for hyperlipidemia, seven trials for impaired glucose tolerance, three trials for obesity, and six trials for combined risk factors. Amongst the included trials with diverse study design, an intervention of $\mathrm{CHM}$ as a supplement to biomedicine and/or a lifestyle intervention was found to be more effective in lowering blood pressure, decreasing blood glucose level, helping impaired glucose tolerance reverse to normal, and/or reducing body weight compared to CHM monotherapy. While no trial reported deaths amongst the CHM groups, some papers do report moderate adverse effects associated with $\mathrm{CHM}$ use. However, the findings of such beneficial effects of $\mathrm{CHM}$ should be interpreted with caution due to the heterogeneous set of complex CHM studied, the various control interventions employed, the use of different participants' inclusion criteria, and low methodological quality across the published studies. The risk of bias of trials identified was largely unclear in the domains of selection bias and detection bias across the included studies.
\end{abstract}

Conclusion: This study showed substantial evidence of varied CHM interventions improving the stroke modifiable risk factors. More rigorous research examining the use of $\mathrm{CHM}$ products for sole or multiple major stroke risk factors are warranted.

Keywords: Chinese herbal medicine, Stroke, Risk factor, Prevention

\section{Background}

Stroke is the second foremost cause of mortality and a leading cause of serious disability worldwide [1]. The incidence of stroke continues to rise due to societal and lifestyle changes and an aging population [2]. More than $90 \%$ of the stroke burden is attributable to its modifiable risk

\footnotetext{
*Correspondence: David.Sibbritt@uts.edu.au

${ }^{3}$ Australian Research Centre in Complementary and Integrative Medicine (ARCCIM), Faculty of Health, University of Technology Sydney, Level 8, Building 10, 235-253 Jones St, Ultimo, NSW 2007, Australia

Full list of author information is available at the end of the article
}

factors such as high blood pressure, high fasting plasma glucose, and high total cholesterol [3]. These stroke risk factors are strongly inter-related and some of them are simultaneous shown as a combined risk factor in people with stroke with higher risk $[4,5]$. Previous research has clearly demonstrated the benefits of treating risk factors such as hypertension, diabetes, hyperlipidemia, obesity, atrial fibrillation, or transient ischaemic attack (TIA) for reducing the prevalence of primary stroke [6, 7]. The treatments of major stroke modifiable risk factors are therefore crucial for informing stroke prevention 
strategies and helping achieve improved quality of life of people with those risk factors and lowered associated health care costs [3].

Chinese herbal medicine (CHM) - therapies and products made from any part of medicinal plants (e.g. leaves and roots) and some non-herb based components (e.g. shells and powdered fossil) [8] - has a history of more than 2500 years with a unique theory of diagnosis and treatment, and is considered a modality of complementary medicine in Western countries [9]. CHM has been increasingly used for a wide range of chronic diseases in China and elsewhere in the form of raw plant materials, powers, capsules, tablets and/or liquids [9-11].

Chinese herbal medicine is a field of health care that may offer potential for addressing related risk factors of stroke [12-14]. Many CHM interventions have long been used for the treatments of some stroke risk factors as individual diseases such as Type 2 diabetes [15], hypertension [8] and obesity [16]. However, the research evidence as to whether specific $\mathrm{CHM}$ therapies or products may be effective in reducing each individual or mixed major risk factors of stroke remains unclear. The aim of this systematic review is to assess and summarize the efficacy and safety of all relevant CHM interventions for people at greatest risk(s) of stroke.

\section{Methods}

\section{Search strategy}

Four key bibliographic databases-MEDLINE, CINAHL/ EBSCO, SCOPUS, and Cochrane Database of Systematic Reviews-were searched in the systematic review.
This review was designed and conducted in accordance with PRISMA (Preferred Reporting Items for Systematic Reviews and Meta-Analyses) guidelines. The stroke modifiable risk factors identified in this systematic review refer to high blood pressure (hypertension), high cholesterol (hyperlipidemia), irregular pulse (atrial fibrillation), TIA, high blood glucose (diabetes and impaired glucose tolerance (IGT), and overweight (obesity). The literature search employed keyword and $\mathrm{MeSH}$ term searches for terms relevant to 'CHM' and terms regarding stroke risk factors (Table 1). The combination of the search results of $\mathrm{CHM}$ and stroke risk factors were identified for screening. To obtain all relevant articles, reference lists of published review papers were also reviewed via Google Scholar.

\section{Study selection}

The inclusion criteria of literature in the systematic review were: peer-reviewed English-language journal articles focusing upon randomized controlled trials (RCTs) or cross-over studies published in the past 20 years (1996-2016), and articles reporting primary data findings examining the efficacy and safety of any type of CHM interventions (e.g. decoction, capsule, granule, power) on one or more major modifiable risk factors of stroke. Exclusion criteria were (1) published RCT protocols of this research area; (2) quasi- or pseudoRCTs (3) studies focusing upon the efficacy and safety of CHM for treating stroke or post-stroke symptoms; (4) studies focusing upon the efficacy and safety of $\mathrm{CHM}$ for treating the complications of the stroke risk factors;

Table 1 Search terms for the systematic review

\begin{tabular}{|c|c|c|}
\hline \multirow{2}{*}{$\begin{array}{l}\text { Chinese herbal medicine } \\
\text { AND }\end{array}$} & \multicolumn{2}{|c|}{$\begin{array}{l}\text { Chinese herbal medicine [MeSH Term \& Keyword] OR Chinese medicine [MeSH Term \& Keyword] OR Chinese } \\
\text { herb* [Title/Abstract] OR Chinese herbal [Title/Abstract] }\end{array}$} \\
\hline & & \\
\hline \multirow[t]{6}{*}{ Stroke risk factors } & High blood pressure & $\begin{array}{l}\text { Hypertension [MeSH Term \& Keyword] OR Blood pressure [MeSH Terms \& Keyword] OR } \\
\text { Hypertens* [Title/Abstract] OR Prehypertens* [Title/Abstract] OR Systolic [Title/Abstract] OR } \\
\text { Diastolic [Title/Abstract] OR }\end{array}$ \\
\hline & High cholesterol & $\begin{array}{l}\text { Cholesterol [MeSH Term \& Keyword] OR Triglycerides [MeSH Term \& Keyword] OR Dyslipi- } \\
\text { demia [MeSH Term \& Keyword] OR Epicholesterol [Title/Abstract] OR HDL [Title/Abstract] } \\
\text { OR LDL [Title/Abstract] OR Triglyceride* [Title/Abstract] OR Hyperlipidem* [Title/Abstract] } \\
\text { OR Lipidem* [Title/Abstract] OR }\end{array}$ \\
\hline & Irregular pulse & $\begin{array}{l}\text { Cardiac arrhythmias [MeSH Terms \& Keyword] OR Atrial fibrillation [MeSH Terms \& Keyword] } \\
\text { OR Dysrhythmia* [Title/Abstract] OR Cardiac arrhythmia* [Title/Abstract] OR }\end{array}$ \\
\hline & Transient ischaemic attack & $\begin{array}{l}\text { Transient ischaemic attack [MeSH Terms \& Keyword] ORTransient ischaemic attack* [Title/ } \\
\text { Abstract] OR }\end{array}$ \\
\hline & High blood glucose & $\begin{array}{l}\text { Diabetes [MeSH Terms \& Keyword] OR Mellitus [MeSH Terms \& Keyword] OR Impaired glucose } \\
\text { tolerance [MeSH Terms \& Keyword] OR Diabet* [Title/Abstract] OR NIDDM [Title/Abstract] } \\
\text { OR IDDM [Title/Abstract] ORT2DM [Title/Abstract] OR *insulin* [Title/Abstract] OR Glucose } \\
\text { [Title/Abstract] OR }\end{array}$ \\
\hline & Overweight & $\begin{array}{l}\text { Obesity [MeSH Terms \& Keyword] OR Overweight [MeSH Terms \& Keyword] OR Metabolic } \\
\text { syndrome [MeSH Terms \& Keyword] OR Obes* [Title/Abstract] OR Adiposity [Title/Abstract] } \\
\text { OR Adipos* [Title/Abstract] }\end{array}$ \\
\hline
\end{tabular}

* Truncation, refering to all records that have those letters with any ending 
(5) conference abstracts; and (6) publications without abstracts.

\section{Data extraction}

Titles and abstracts of all citations identified in the initial search were imported to Endnote (Version X7) and duplicates removed. Two of the authors screened all the titles/abstracts to identify articles meeting the inclusion and exclusion criteria independently. When consensus was not reached, the full texts of these unclear papers were retrieved and assessed by these two authors. Disagreements were discussed with a third author.

Data were extracted into a pre-determined table (Table 2) and checked for coverage and accuracy by two of the authors. Any differences in data extraction and interpretation were resolved through discussion amongst all authors. Table 2 includes detailed information on study recruitment, participant characteristics, intervention groups, results of primary outcome measures, study limitations, and CHM safety.

\section{Quality assessment}

Two authors independently assessed the methodological quality of the included studies using the Cochrane risk of bias criteria [17]. The characteristics of RCTs that might be related to selection bias (random sequence generation and allocation concealment), performance bias (blinding of participants and personnel), detection bias (blinding of outcome assessment), attrition bias (incomplete outcome data), reporting bias (selective outcome reporting), and other bias were evaluated. Disagreements regarding the risks of bias of some studies were resolved through discussion amongst these two authors (Table 3).

\section{Results}

The systematic review reported in this paper has been registered on the PROSPERO (International prospective register of systematic reviews, \#CRD42017060107). The PRISMA flowchart of literature search and study/ article selection has been shown in Fig. 1. A total of 2377 papers were identified (2374 via database searches and three additional papers via Google Scholar). After removing duplicates, a total of 2065 papers remained for review. From amongst these, 70 manuscripts were identified for full review following title and abstract screening. Further screening of the full texts identified 46 publications (reporting on $46 \mathrm{RCTs}$ ) as eligible for final inclusion in the systematic review. Twelve of the included articles report on the efficacy of CHM for hypertension (1340 participants), 10 for diabetes (2004 participants), eight for hyperlipidaemia (997 participants), seven for IGT (1805 participants), three for obesity (329 participants), and six for the combination of several stroke risk factors (420 participants). No manuscript reported on a trial investigating the efficacy of CHM interventions for the stroke risk factor of transient ischemic attack or atrial fibrillation as a primary outcome. The characteristics of included studies with regards to the CHM interventions for hypertension, diabetes, hyperlipidaemia, IGT, obesity, and combined stroke risk factors are summarized in Table 2.

\section{Hypertension}

Eight RCTs were focused upon primary (essential) hypertension [18-25], one with isolated systolic [26], one with elder polarized hypertension [27], and two with hypertension and related cardiovascular diseases [28, 29]. Of the 12 RCTs on CHM for hypertension, 11 RCTs originated from China [18-22, 24-29]. Amongst the hypertension-focused RCTs, one RCT compared 'CHM, biomedicine plus lifestyle' intervention with 'biomedicine plus lifestyle' intervention [27] and showed significant decreased systolic blood pressure (SBP) before and after treatment of both intervention groups and a similar effect on controlling SBP between these two groups after treatment. Another two RCTs compared two different CHM interventions using different inclusion criteria of people with hypertension $[19,21]$-these studies both reported a significant decrease of SBP and diastolic blood pressure (DBP) via all the CHM interventions examined with higher effective rate of treatments in the $\mathrm{CHM}$ groups than those in the control groups. Another three RCTs compared 'CHM' interventions with 'biomedicine' interventions and employed consistent inclusion criteria regarding SBP $(140-179 \mathrm{mmHg})$ and DBP (90-109 $\mathrm{mmHg}$ ) of participants, reporting a statistically significant decrease of SBP and DBP before and after treatment of both groups and a similar effect on controlling SBP and DBP between these two groups after treatment $[18,22,24]$. Another six RCTs compared 'CHM plus biomedicine' interventions with 'biomedicine alone' or 'biomedicine plus placebo' interventions [20, 23, 25, $26,28,29]$. It is noteworthy that two of these six trials $[20,28]$ examined the efficacy of the same CHM products (Xuezhikang capsule) at different dose levels, demonstrating a significant decrease of SBP and DBP before and after treatment of both intervention groups and a silimar effect on SBP and DBP control between these two groups after treatment. Also amongst these six RCTs, three were three-armed RCTs which compared either 'CHM plus biomedicine' intervention versus 'biomedicine/no intervention', 'CHM' interventions versus ' $\mathrm{CHM}$ plus biomedicine' or 'placebo plus biomedicine' intervention, or two types of preparations of a ' $\mathrm{CHM}$ plus biomedicine' intervention versus 'placebo plus biomedicine' intervention $[25,26,28]$, showing inconsistent findings 












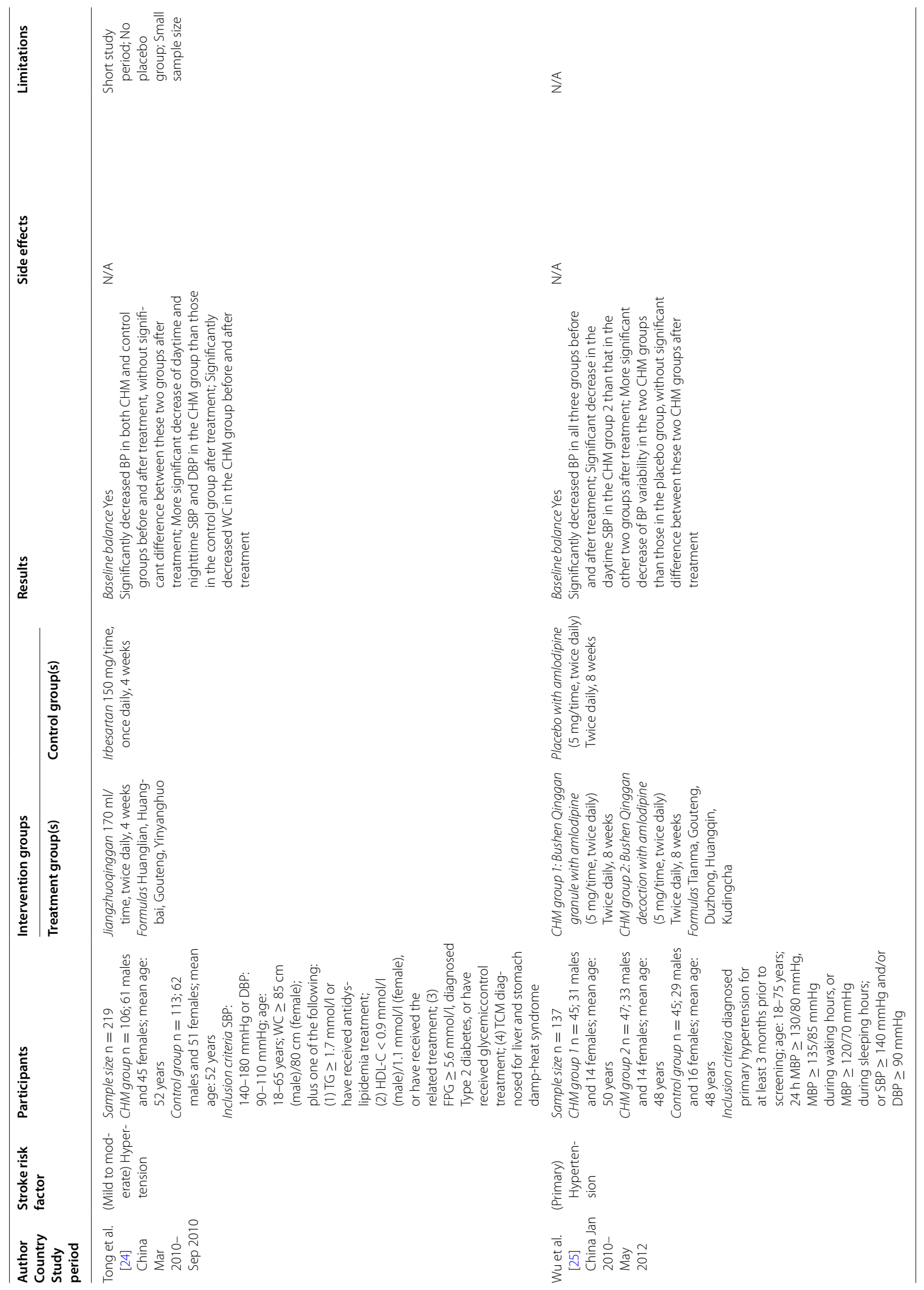




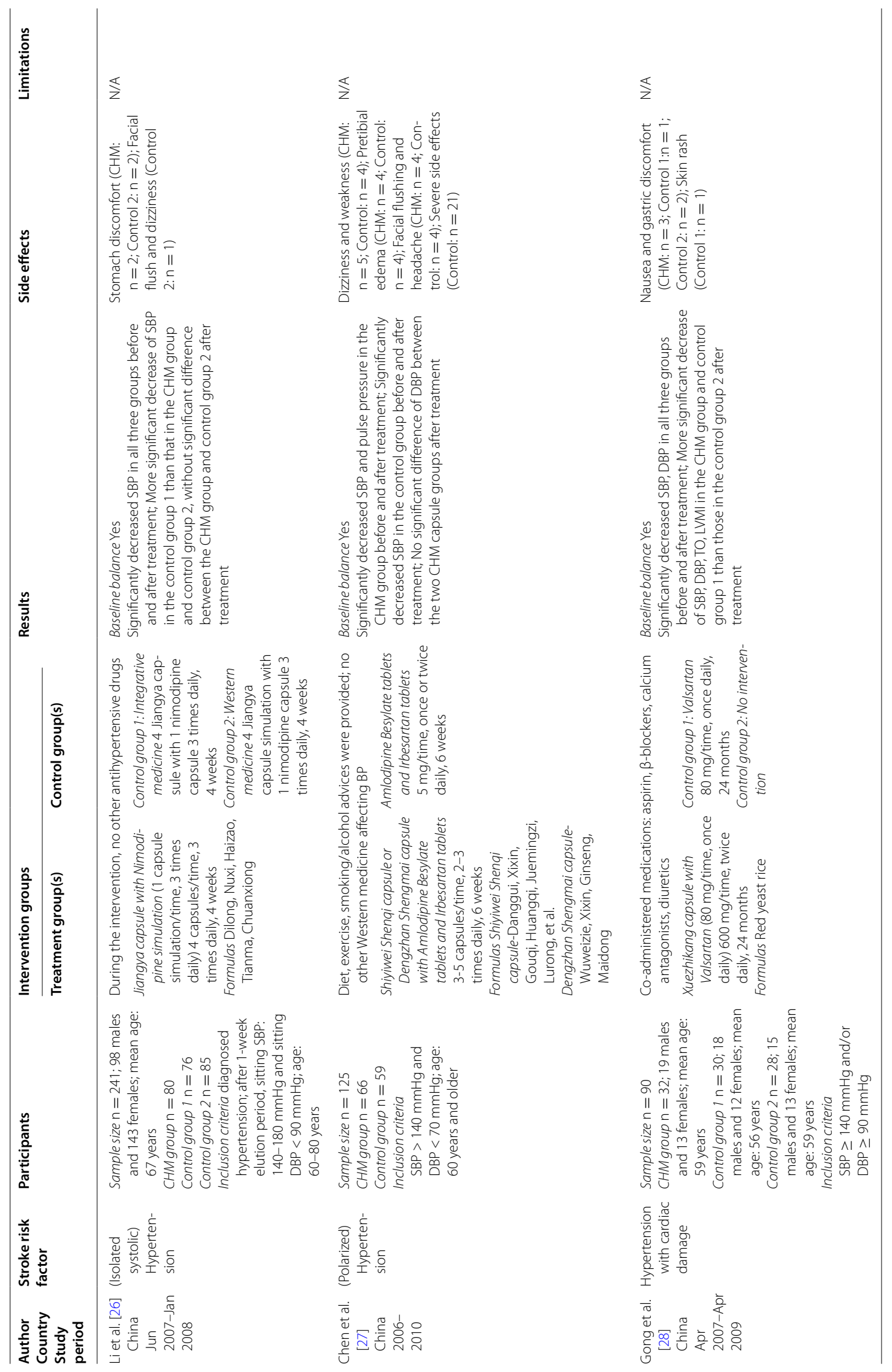




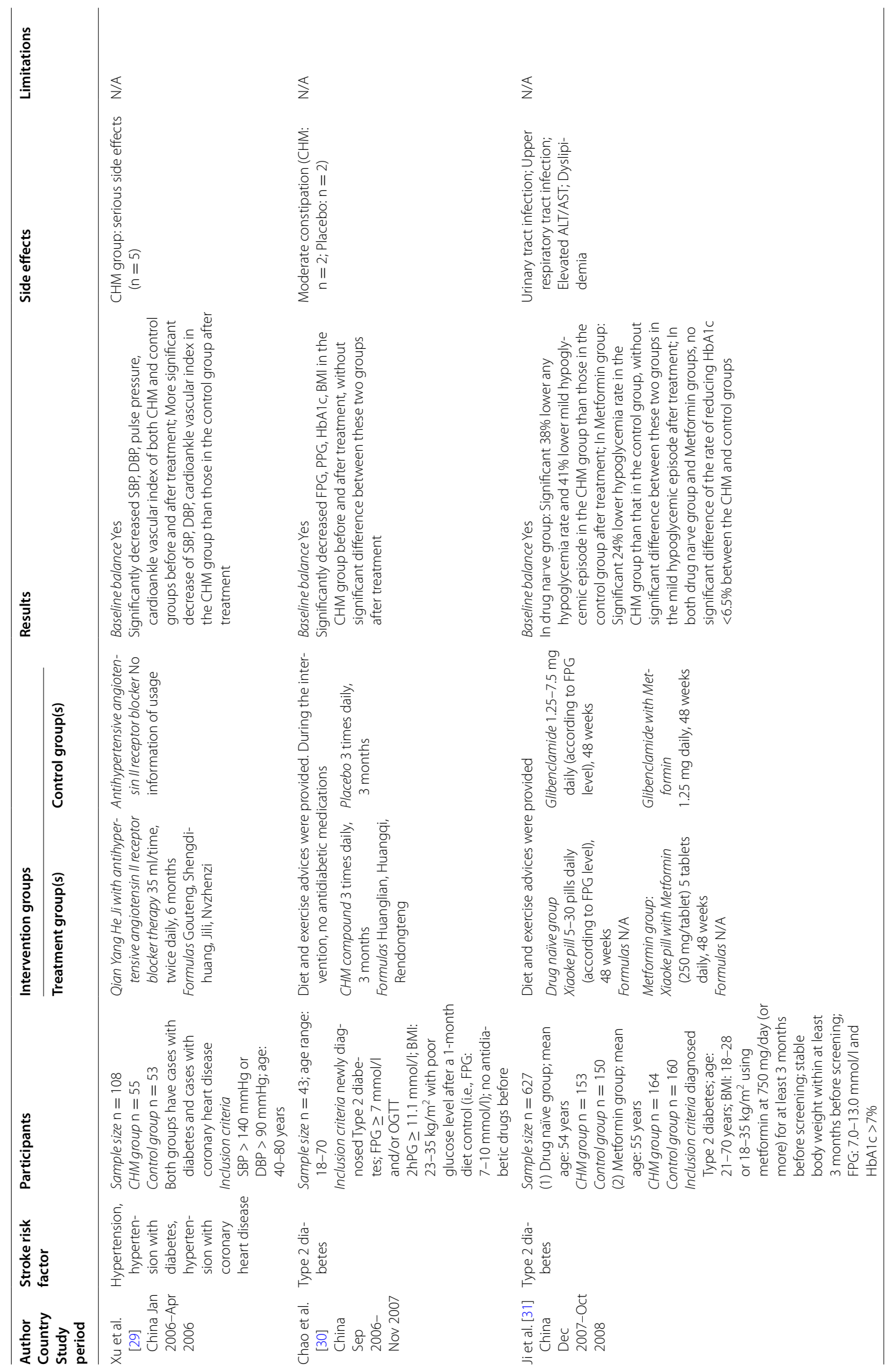




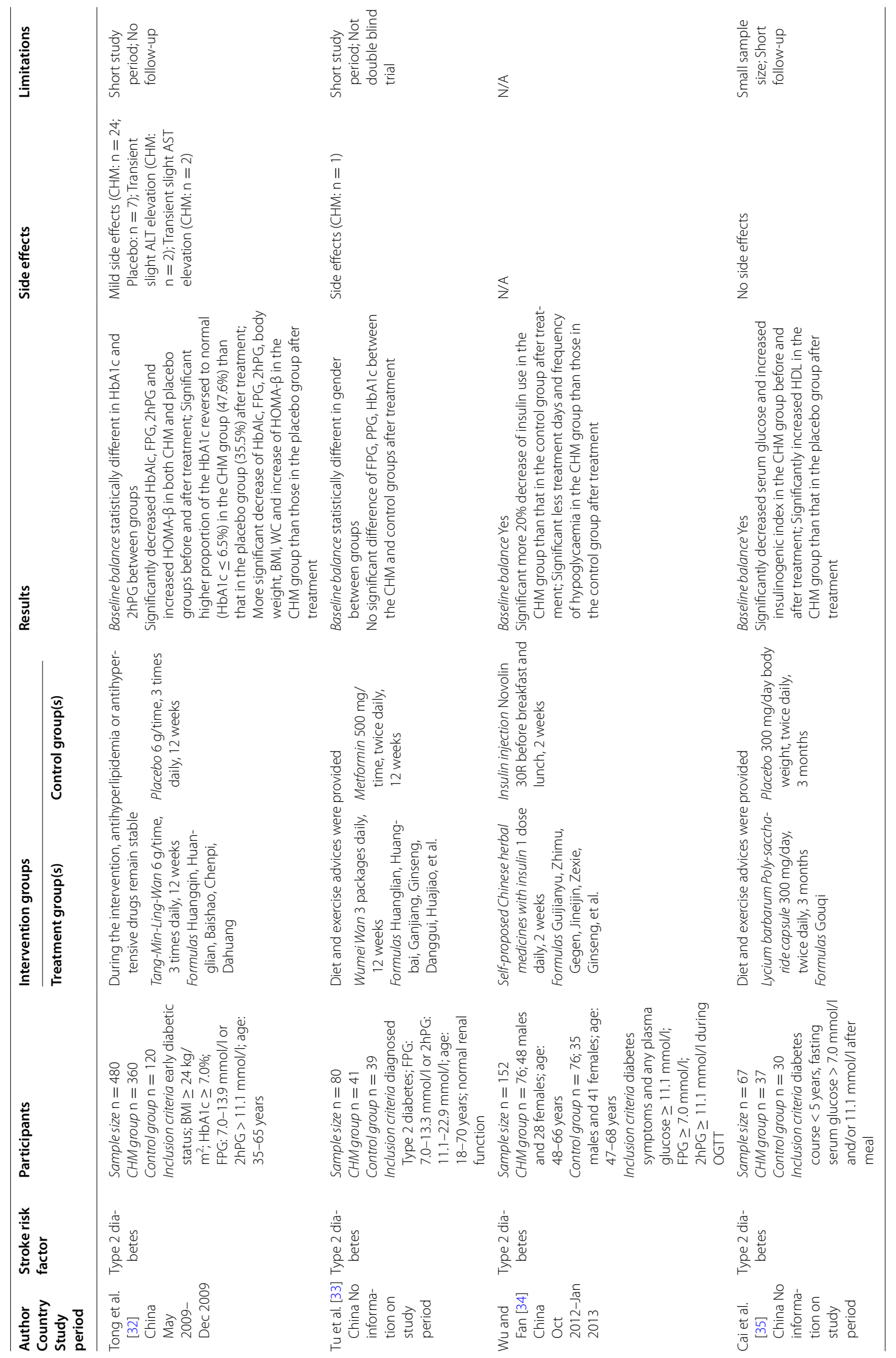




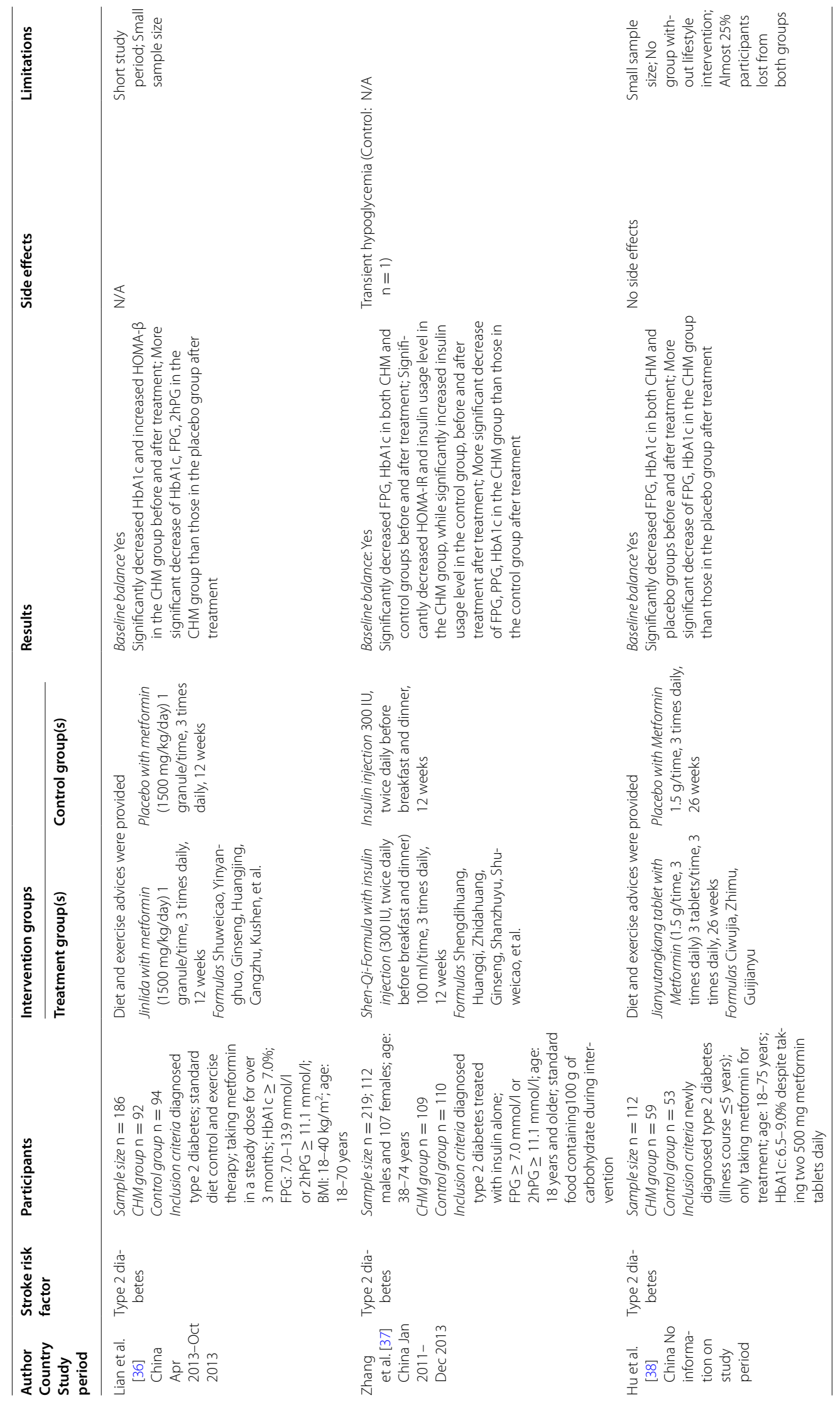




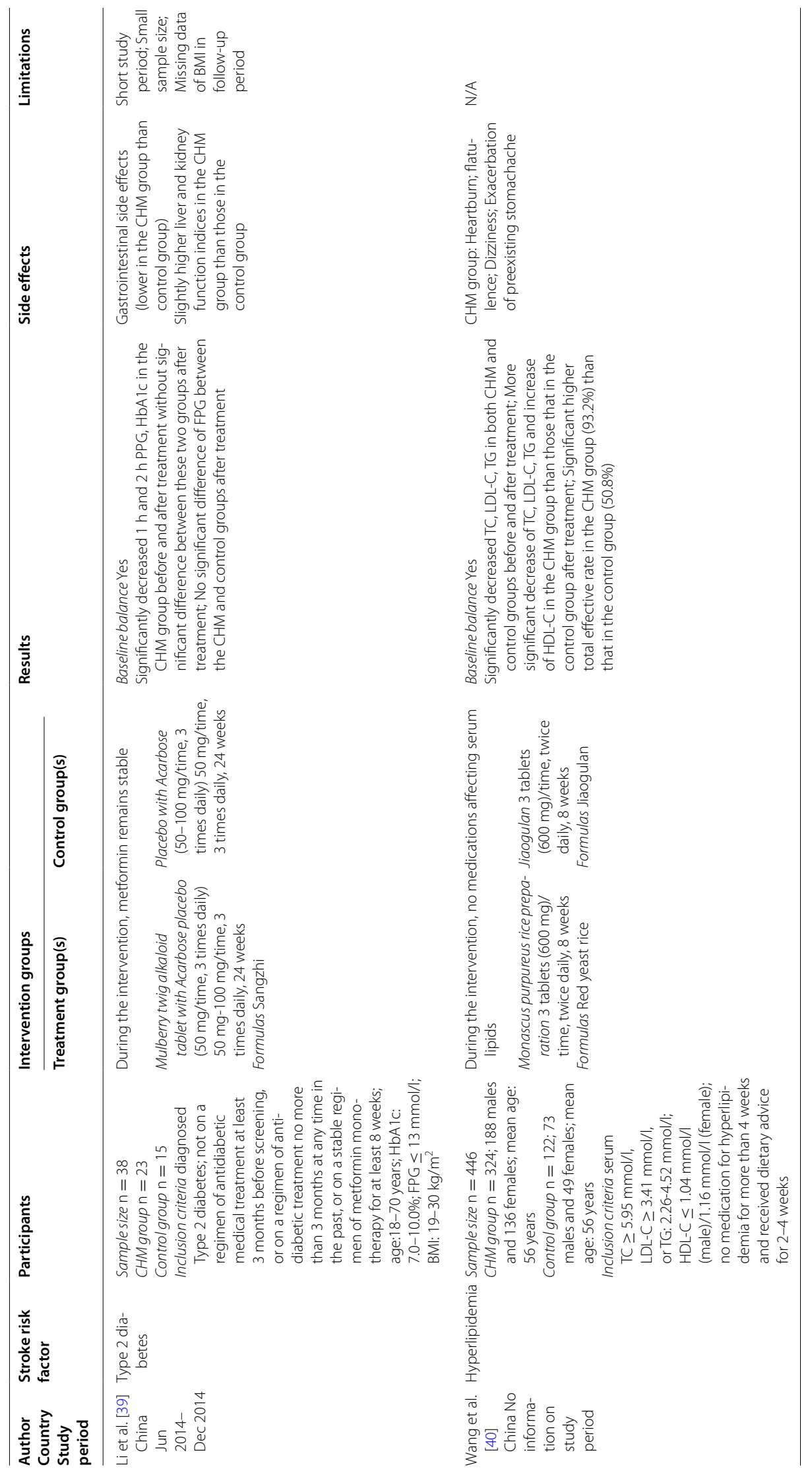




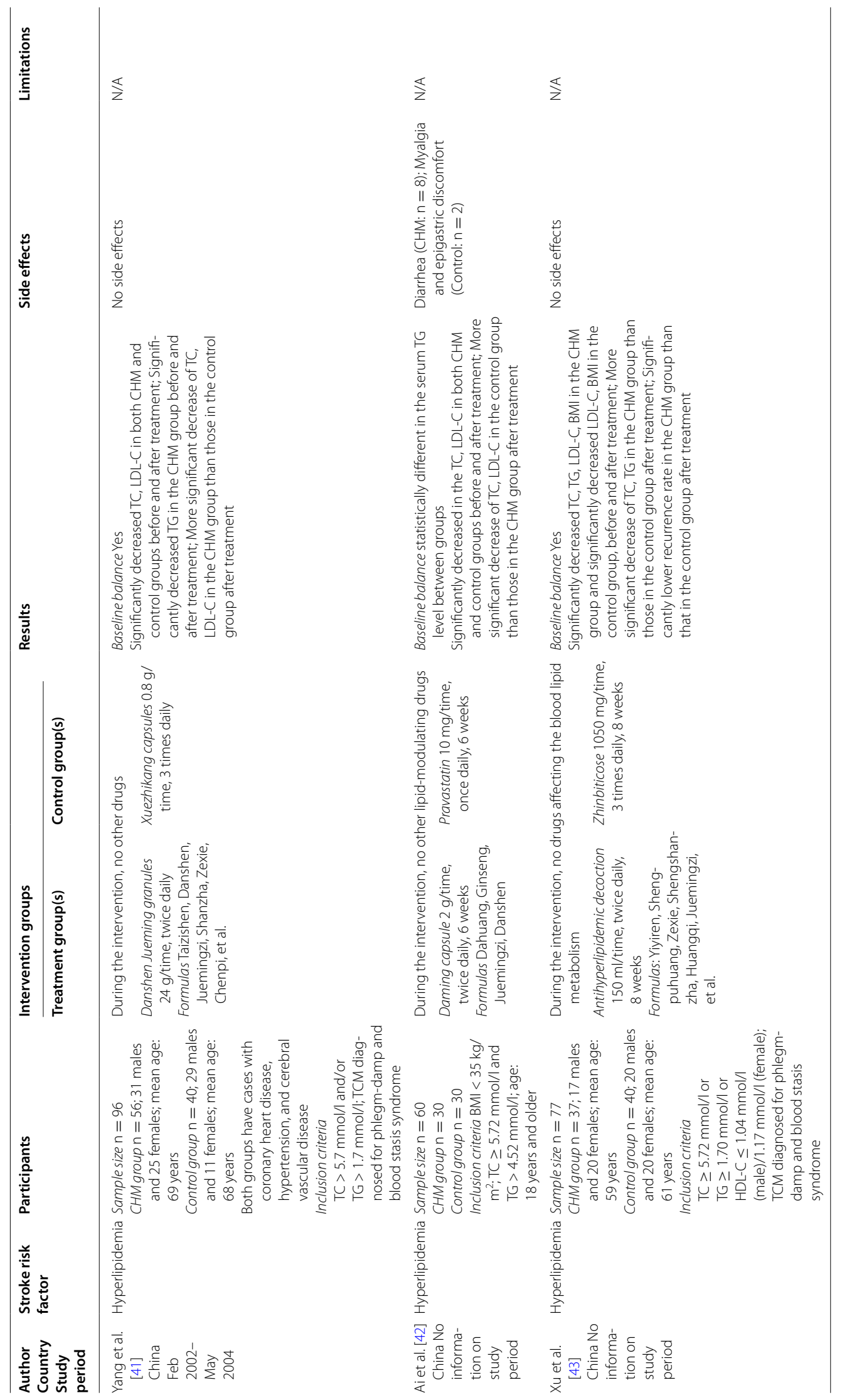




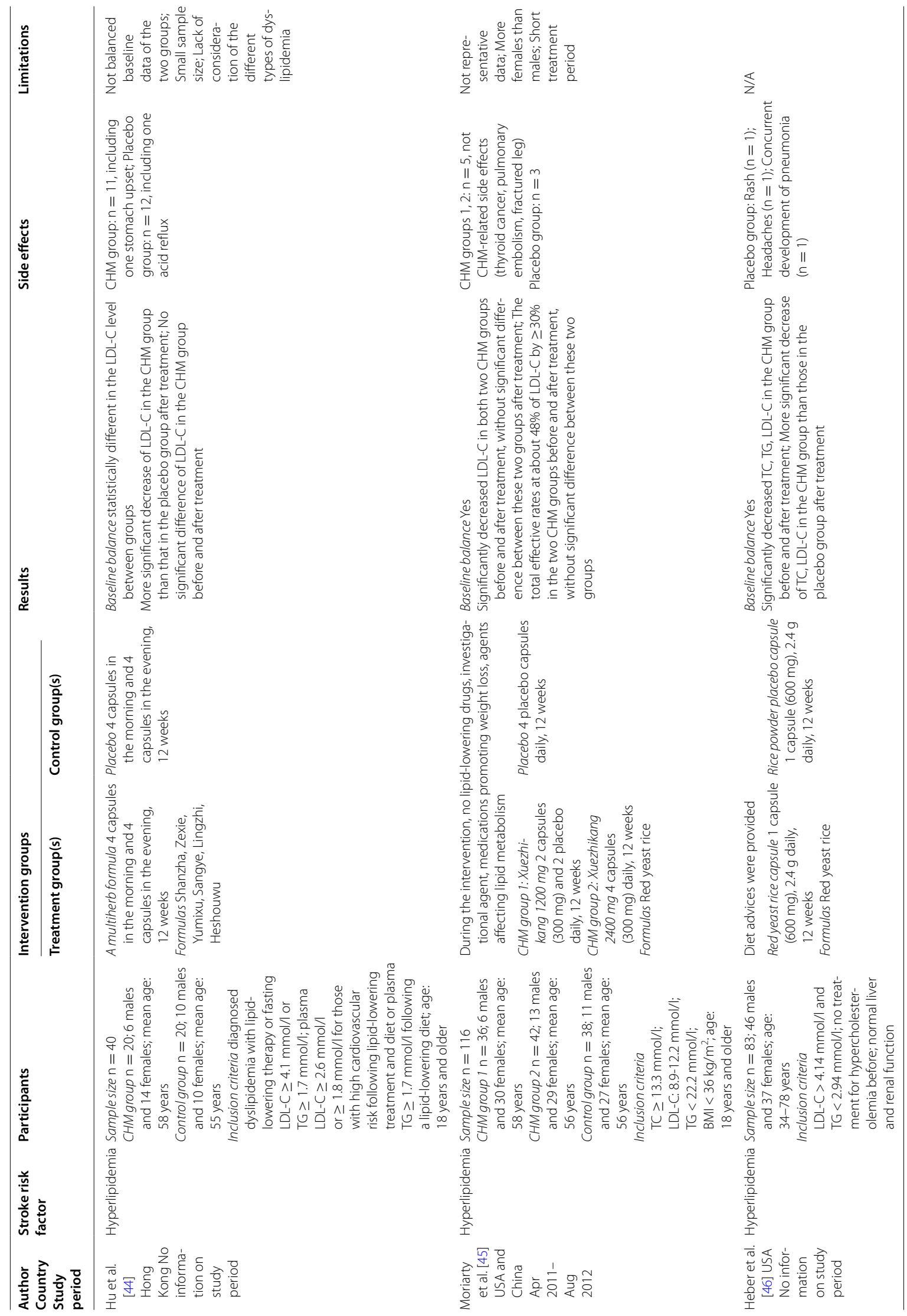




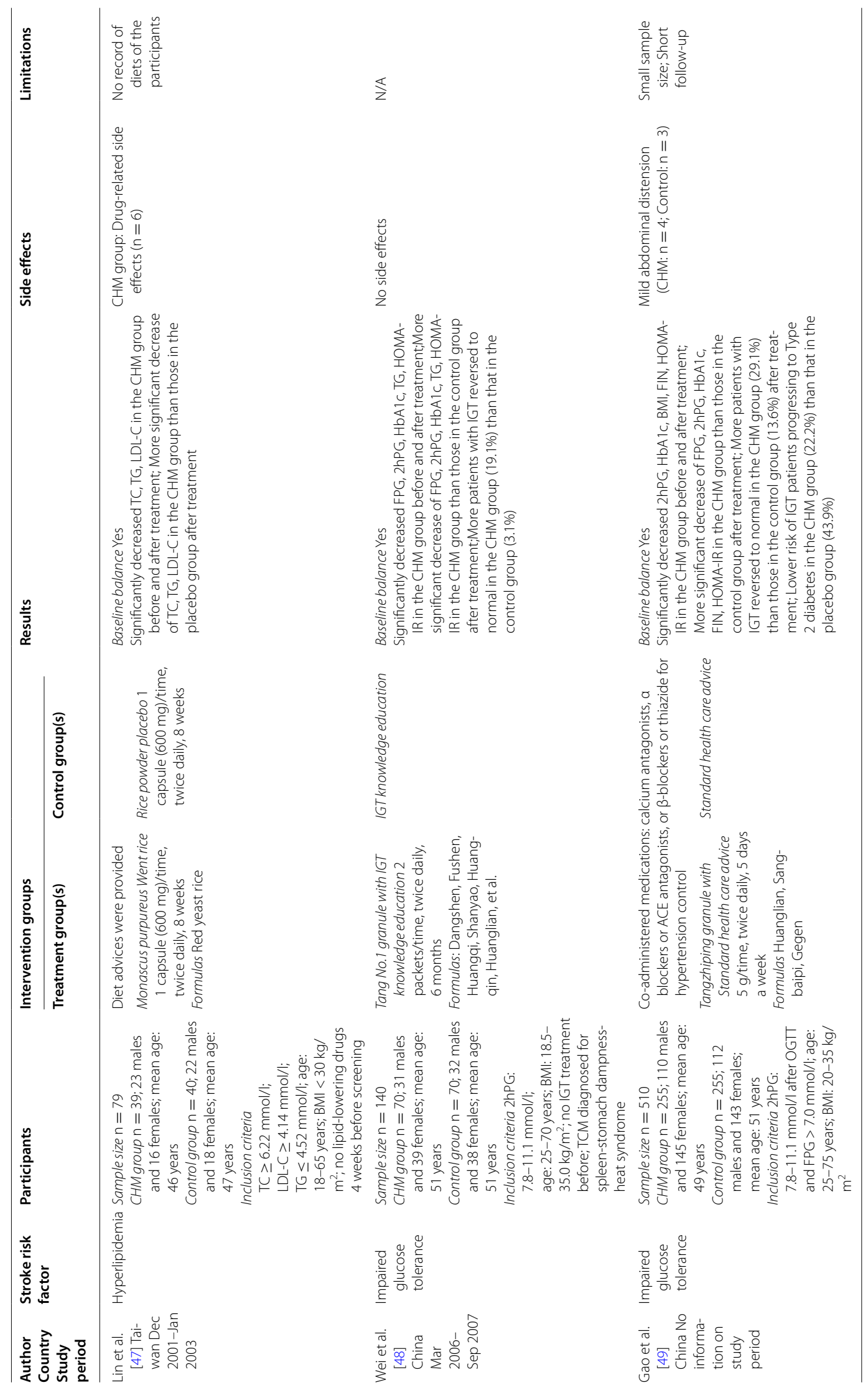




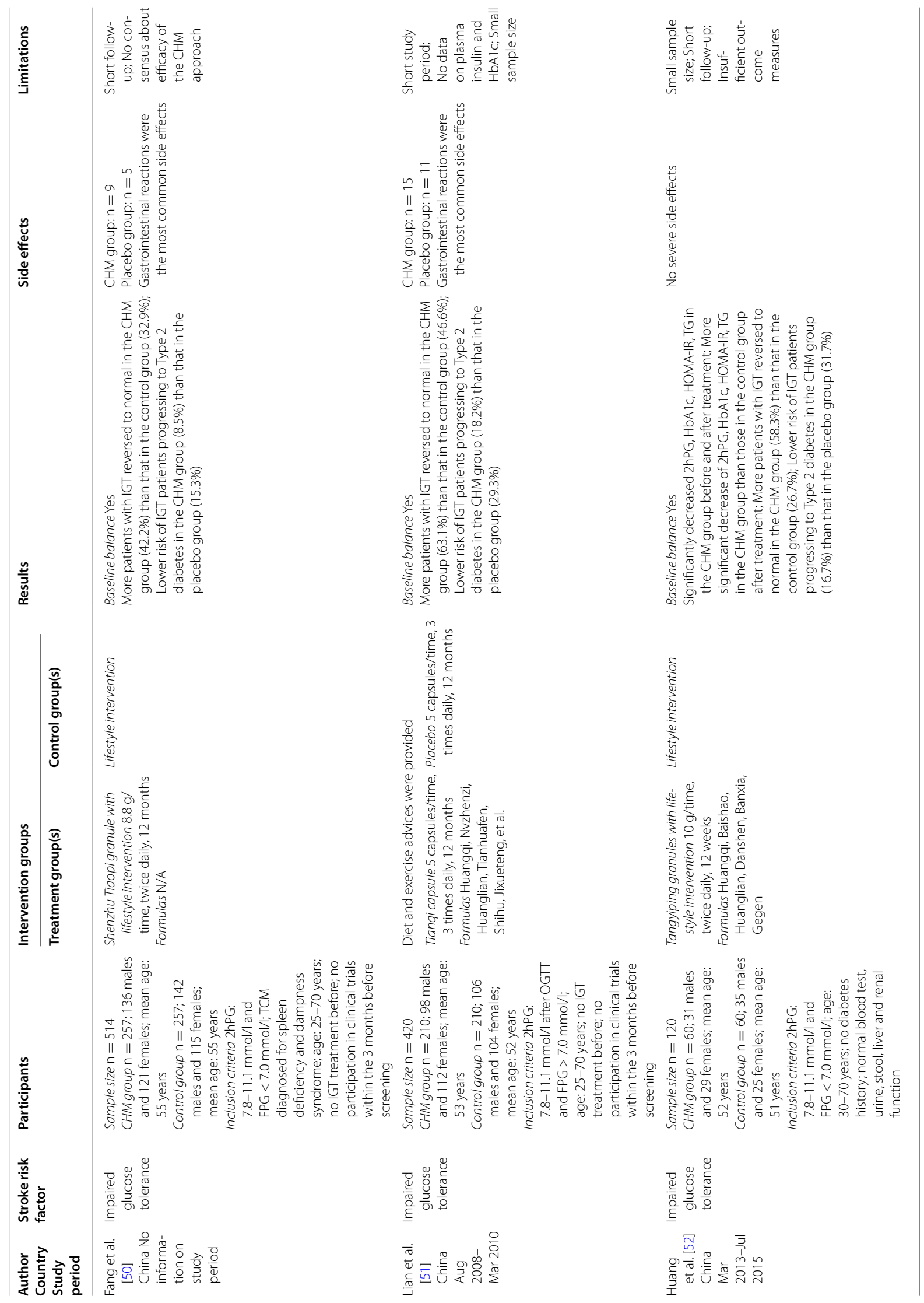




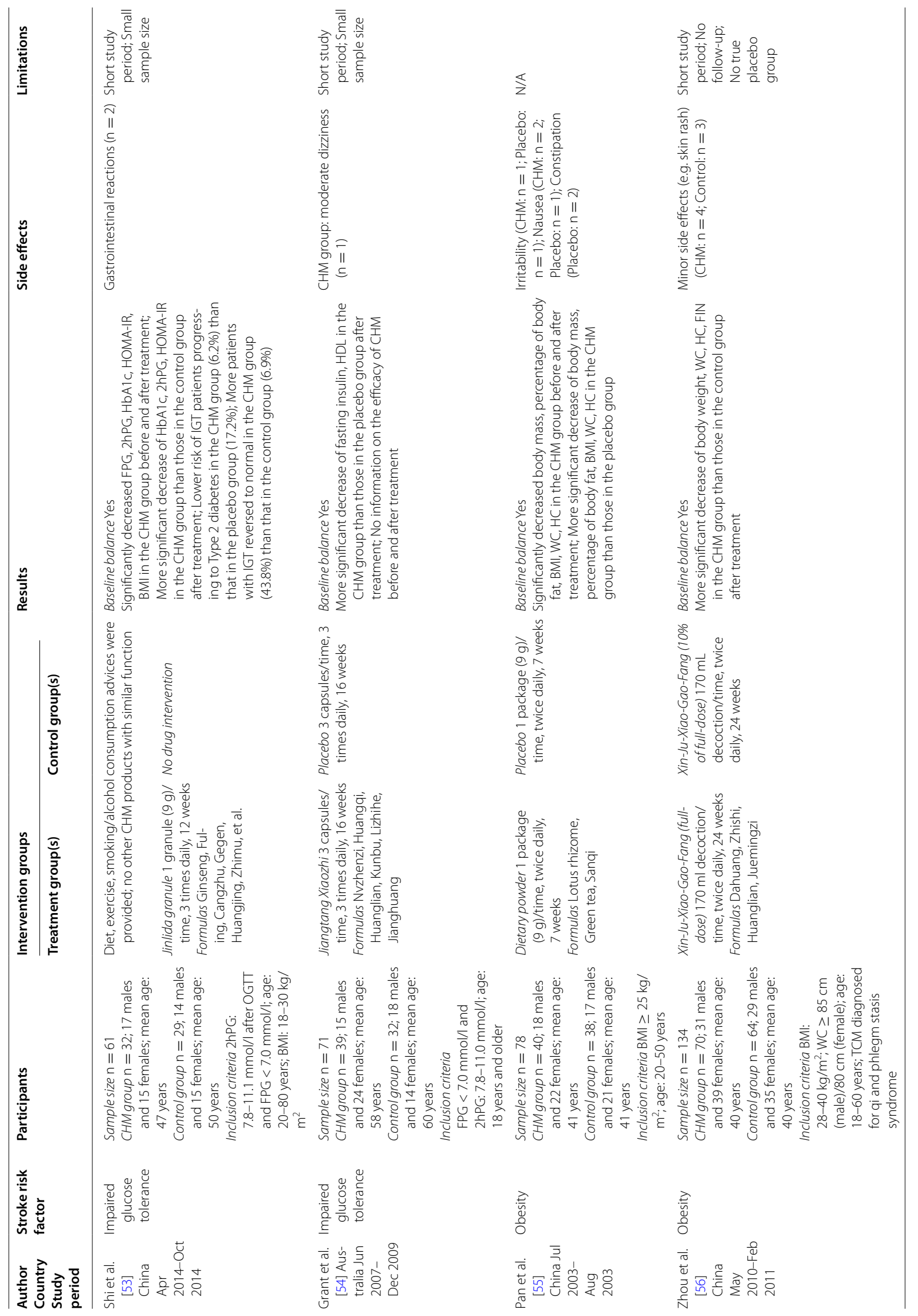




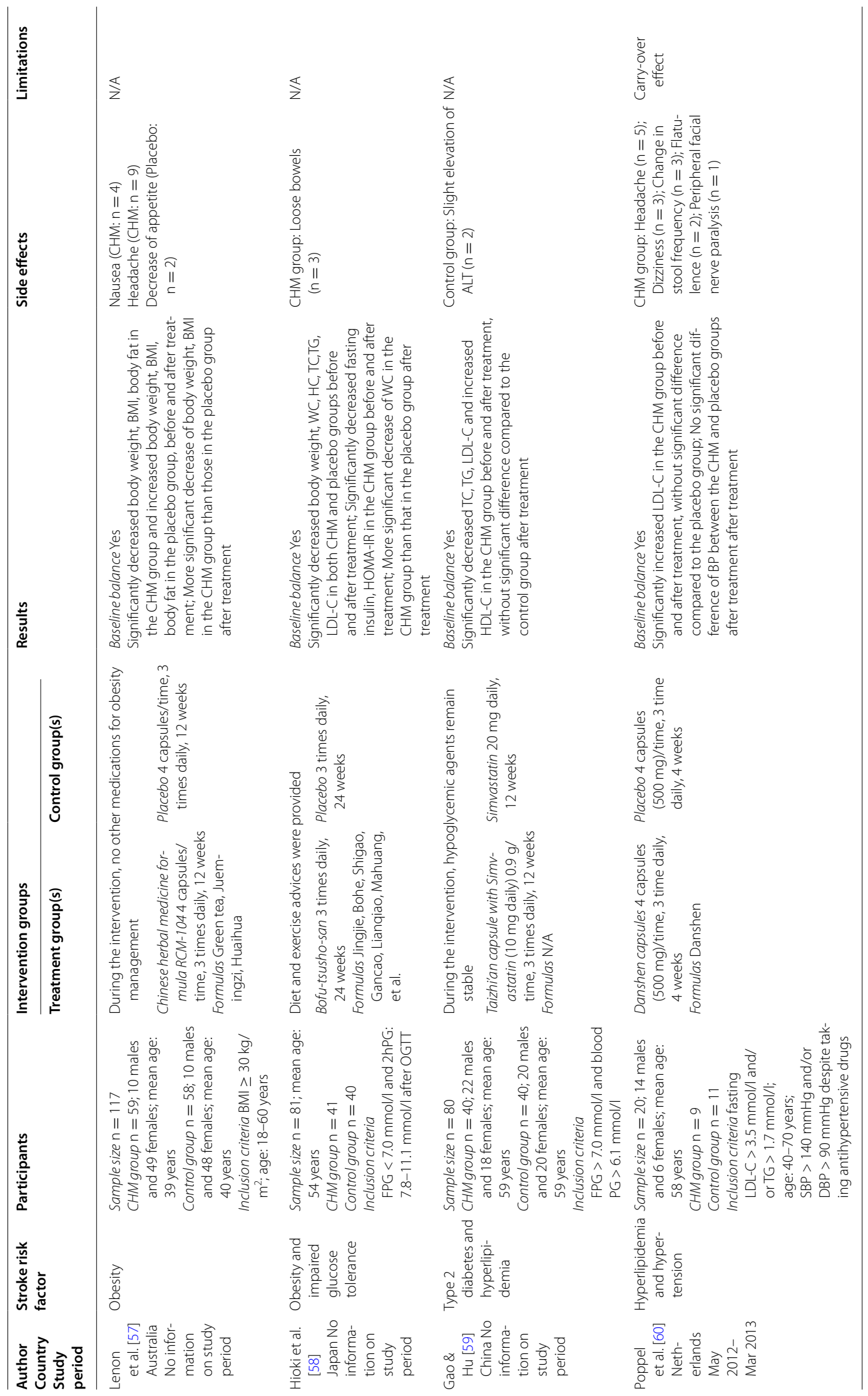




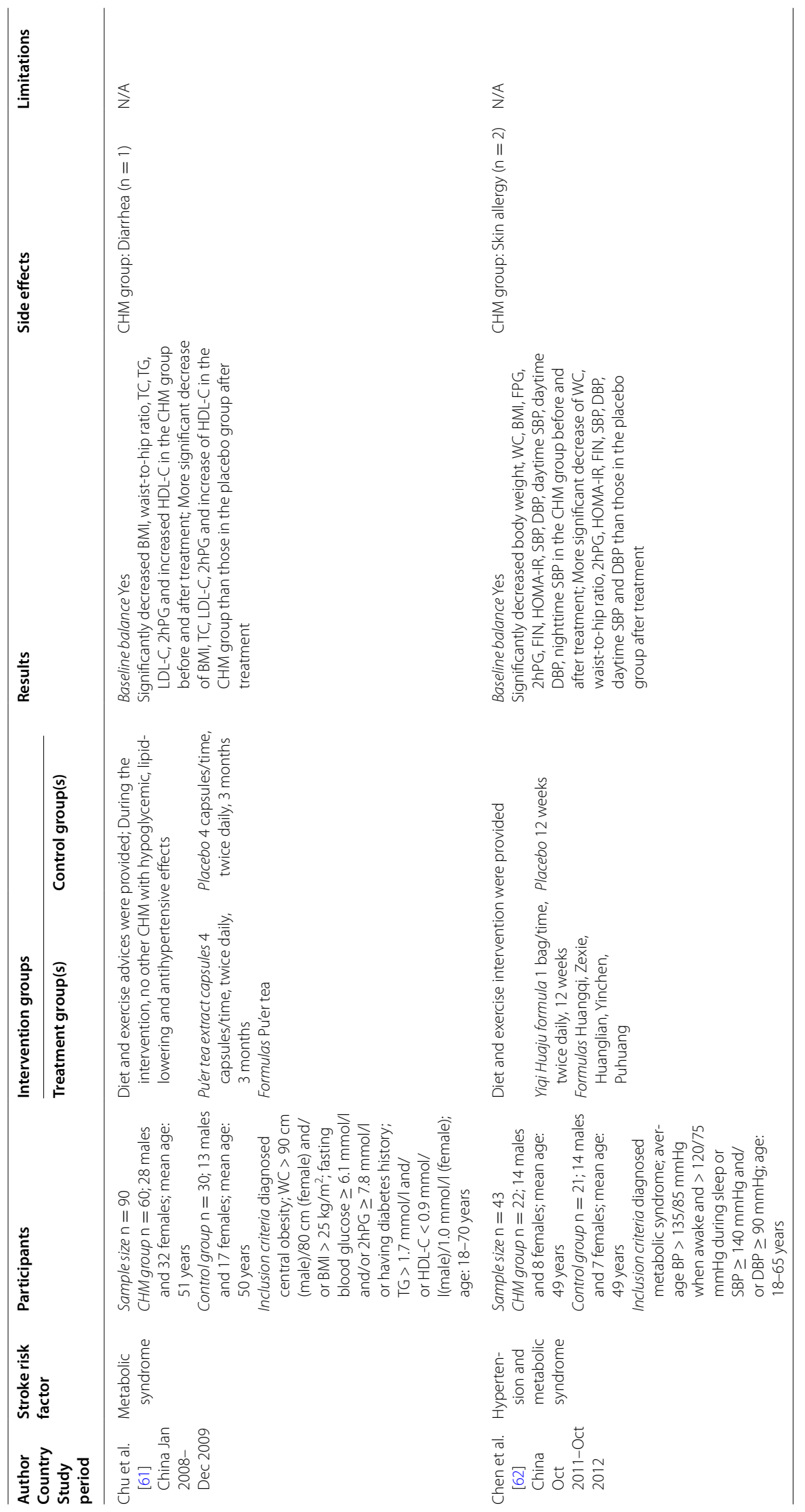




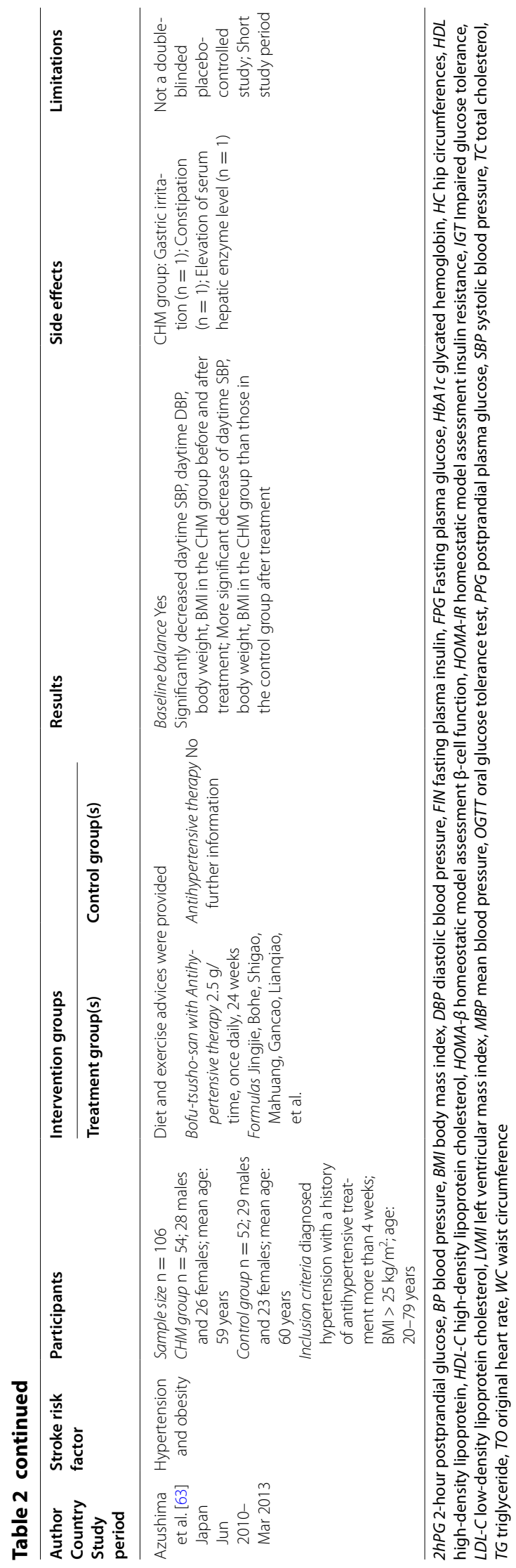




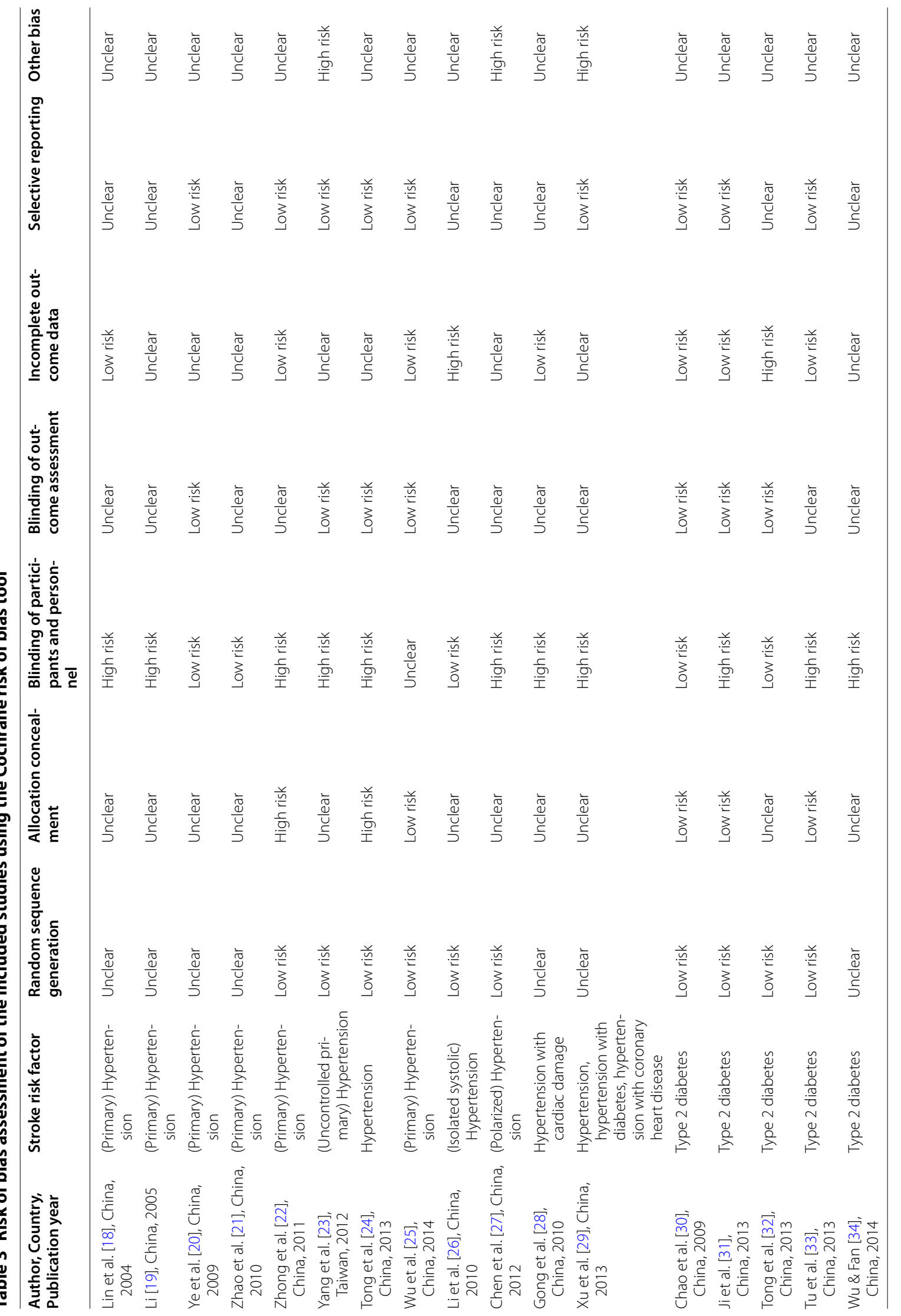




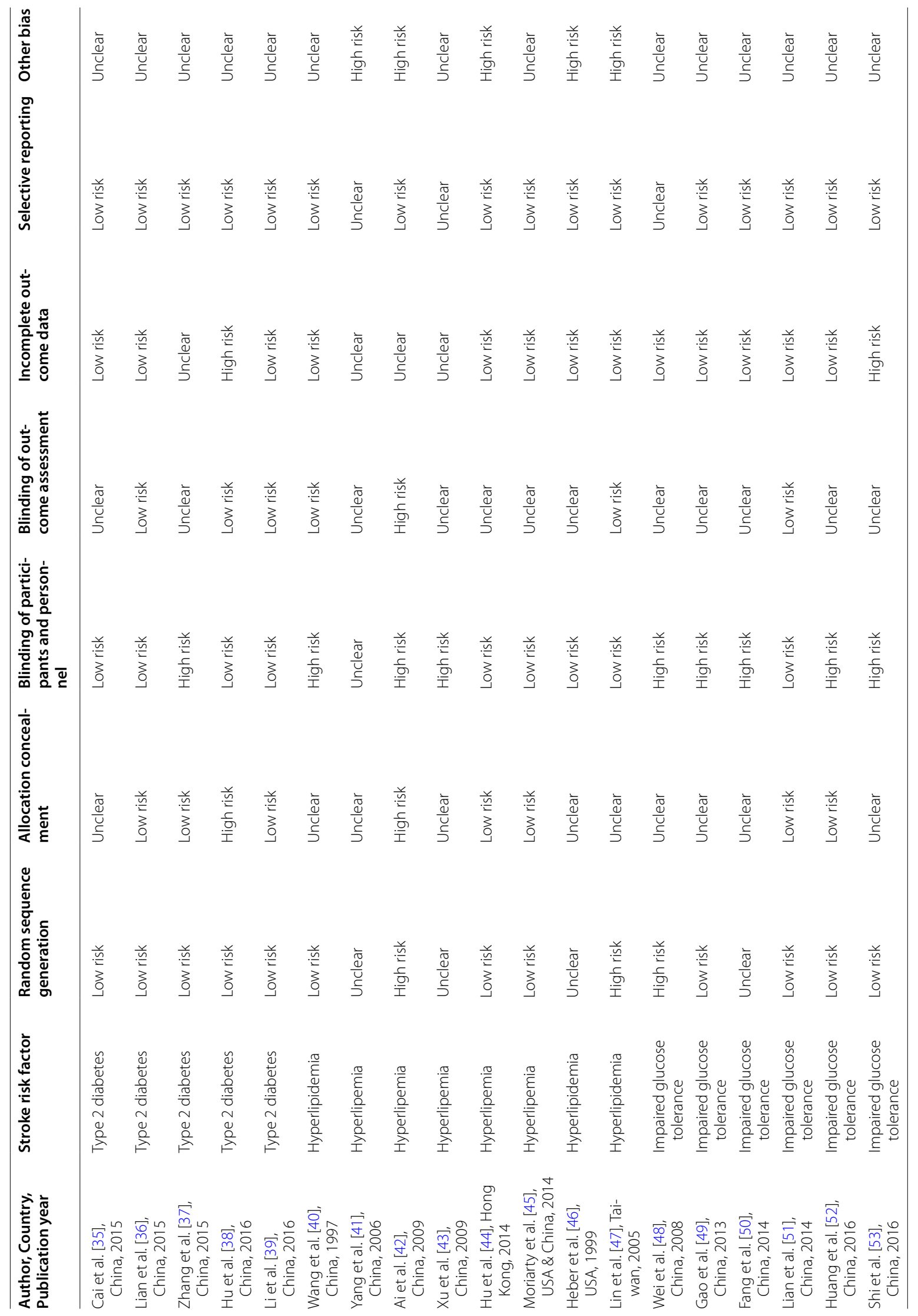




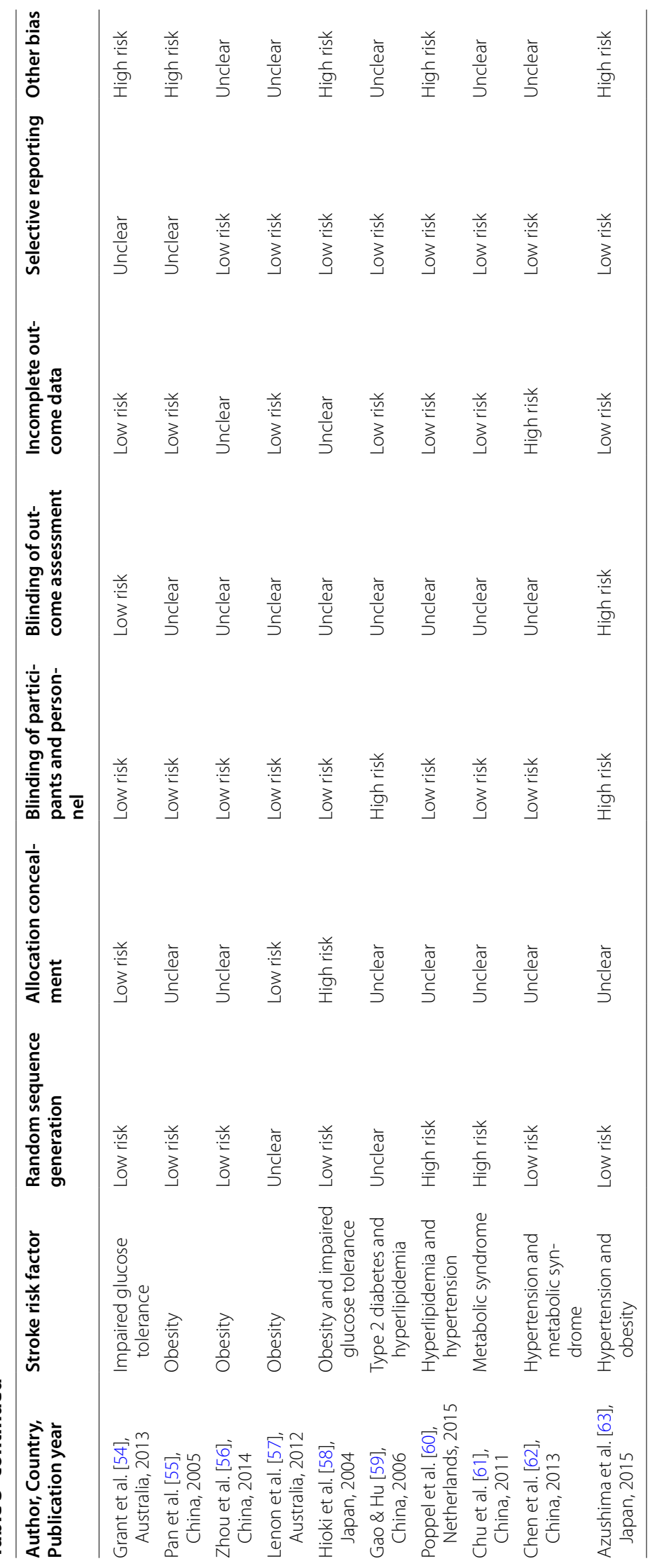






Records identified through database searching $(n=2374)$

- Medline $\quad(n=1308)$

- Scopus $(\mathrm{n}=329)$

- CINAHL/EBSCO $(\mathrm{n}=79)$

- Cochrane library $(\mathrm{n}=658)$



Additional records identified through other sources $(n=3)$

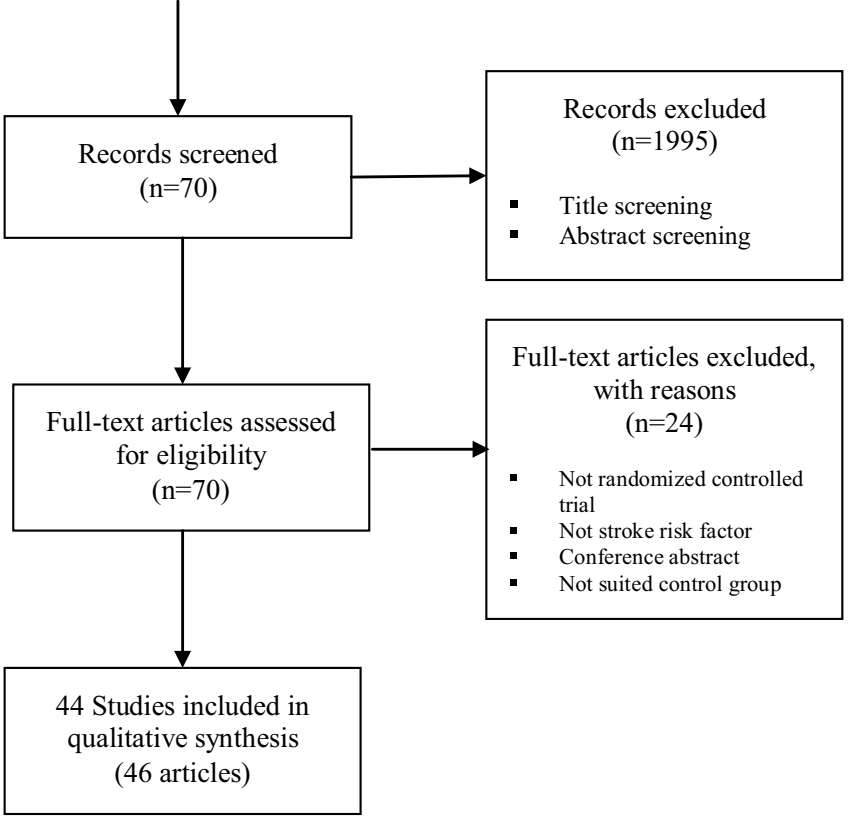

Fig. 1 PRISMA flowchart of literature search and study selection

with regards to the decrease of SBP or DBP amongst the three groups after treatment. Gouteng (钩藤) [18, 19, 21, 24, 25, 29] and Tianma (天麻) [18, 22, 25-27] were the most frequently used Chinese herbs in the hypertensionfocused RCTs included, and all the CHM interventions using Gouteng and/or Tianma reported significant prepost effectiveness regarding the decrease of SBP (and/ or DBP) level. Also, Gouteng was the principal CHM formula constituent amongst four out of six hypertensionfocused RCTs presenting between-group effectiveness of the investigated CHM interventions on the decrease of SBP (and/or DBP) levels compared to control interventions [21, 24, 25, 29]. In addition, the sample size of hypertension-focused RCTs ranged from 55 to 219. Six hypertension-focused RCTs did not provide the age and gender profile of the participants in either CHM group or control group [20, 22, 23, 26, 27, 29]. The duration of the hypertension-focused trials ranged from 2 weeks to 24 months, with the majority of trials conducted between 4 and 12 weeks.

Eight hypertension-focused RCTs reported safetyrelated information and no deaths were noted $[18,19,21$, 23, 26-29]. One trial reported five cases of serious side effects of the 'CHM plus biomedicine' intervention group [29]. One trial (sample: 55) reported 13 mild side effects in the 'CHM plus biomedicine' intervention group and 15 in the 'placebo plus biomedicine' control group [23]. Only two of the papers reporting results from hypertensionfocused RCTs listed any study limitations including small sample size and short study period [23, 24]. As for risk of bias in the hypertension-focused RCTs, three papers provided information on the allocation concealment $[22,24$, 
$25]$ and four on the blinding of outcome assessment [20, 23-25]. Additionally, only three trials reported doubleblinding of participants and personnel involved [20, 21, 26].

\section{Diabetes}

All of the 10 included diabetes-focused RCTs were focusing upon patients diagnosed with Type 2 diabetes mellitus and all these RCTs were conducted in China [30-39]. Amongst the $10 \mathrm{RCTs}$ examining the efficacy of $\mathrm{CHM}$ on controlling the glucose level of patients with diabetes, four RCTs compared 'CHM' intervention to 'placebo' [32], 'CHM plus biomedicine' intervention to 'placebo plus biomedicine' intervention [39], and further, 'CHM plus lifestyle' intervention to 'placebo plus lifestyle' intervention [30,35]. These four trials indicated more significant decreased glucose level [e.g. fasting plasma glucose (FPG), 2-hour postprandial glucose (2hPG), glycated hemoglobin (HbA1c)] by using CHM products when compared to the placebos after treatment, while this significant between-group variance in the decrease of glucose level showed no statistical significance when both $\mathrm{CHM}$ interventions and placebos were used concurrently with biomedicine or lifestyle intervention. Also amongst these 10 diabetes-focused RCTs, 'CHM plus biomedicine' intervention was compared to 'biomedicine' intervention, showing a more significant decrease of insulin usage by the CHM plus biomedicine treatment after treatment [34]. Also, after treatment, 'CHM, biomedicine plus lifestyle' interventions were found to achieve a more significant decrease of FPG, HbA1c, or hypoglycemia when compared to either 'biomedicine plus lifestyle' intervention [31,37] or 'placebo, biomedicine plus lifestyle' intervention [36, 38]. Of the nine diabetes-focused RCTs providing CHM formulas, Huanglian (黄连) was the most common Chinese herb [30, 32-34, 36], followed by Ginseng (人参) [33, 34, 36, 37], Shanzhuyu (山茱莫) [34, 36, 37], Dahuang (大黄) [32, 34, 37], and Huangqi ( 黄芪) [30, 34, 37]. The CHM interventions examined in three out of five diabetes-focused RCTs, showing significant between-group effectiveness on the decrease of glucose level, indicated that the combination of these five commonly used Chinese herbs played a vital role for the efficacy of type 2 diabetes management [34, 36, 37]. All diabetes-focused RCTs defined inclusion criteria of diabetes based on different FPG, 2hPG, and/or HbA1c levels, and all the tested CHM products used in these RCTs were different. The sample size of the diabetes-focused RCTs ranged from 43 to 627 . Only one RCT provided the age and gender profile of participants in the CHM and control groups [35]. The duration of the trials ranged from 2 weeks to 12 months, with the majority of trials conducted between 3-12 months.
Only two diabetes-focused RCTs failed to report safety-related information and no death were noted [34, 36]. The side effects of CHM products reported in the diabetes-focused RCTs are generally moderate, such as constipation, gastrointestinal disorders, and urinary tract infection. However, three diabetes-focused RCTs showed that CHM interventions caused slightly abnormal liver and kidney function after 3, 6, and 12 months, respectively [31, 32, 39]. Six diabetes-focused RCTs have specified their study limitations, with a short study period being the most common issue, followed by small sample size and no/short follow-up period [32, 33, 35, 36, 38, 39]. As for risk of bias of the diabetes-focused RCTs, one trial failed to use the random sequence generation method [34], three trials did not report information on allocation concealment [32, 34, 35], four trials failed to apply a double-blinding method [31, 33, 34, 37], and four trials did not provide details on the blinding outcome assessment $[33-35,37]$.

\section{Hyperlipidemia}

Half of the eight RCTs on CHM for the treatment of hyperlipidemia originated from China [40-43]. Amongst the hyperlipidemia-focused RCTs, two compared 'CHM' interventions with 'biomedicine' interventions [42, 43], two compared different 'CHM' interventions [40, 41], two compared 'CHM' interventions with 'placebos' [44, $45]$ and two compared 'CHM plus lifestyle' interventions with 'placebo plus lifestyle' interventions [46, 47]. Although the inclusion criteria of people with hyperlipidemia shown in the included hyperlipidemia-focused RCTs are limited to the total cholesterol (TC), triglyceride (TG), low-density lipoprotein cholesterol (LDLC), high-density lipoprotein cholesterol (HDL-C), and/ or body mass index (BMI) levels, the threshold value of these indices are diverse across the RCTs. It is worth noting that Monascus purpureus rice preparation (Xuezhikang capsule in Chinese) of which the main ingredient is red yeast rice, was tested in four hyperlipidemia-focused RCTs [40, 45-47]. The effects of the red yeast rice products are not consistent across these four RCTs. When the 'red yeast rice product plus lifestyle' intervention was compared with 'placebo plus lifestyle' intervention, a more significant decrease of TC and LDL-C was found in the red yeast rice product group after treatment. However, there was no significant improvement in TC or LDL-C amongst those receiving the red yeast rice product alone when compared to placebo alone. Amongst the rest four hyperlipidemia-focused RCTs, Danshen (丹参) [41-43], Juemingzi (决明子) [41-43], Zexie (泽泻) [41， 43, 44], and/or Shanzha (山楂) [41, 43, 44] were the main constituents of the CHM formulas examined and three of these trials reported the significant between-group 
effectiveness of the investigated $\mathrm{CHM}$ interventions on the decrease of TC, LDL-C, and/or TG levels [41, 43, 44] compared to control interventions. The sample size of the hyperlipidemia-focused RCTs ranged from 40 to 446 . Only two hyperlipidemia-focused RCTs did not provide the age and gender profile of the participants in CHM and control groups $[42,46]$. The duration of the trials ranged from 6 weeks to 12 months while one trial did not specify the study period.

All hyperlipidemia-focused RCTs reported safetyrelated information and no deaths were noted. Three trials specified their side effects in the CHM intervention groups, including heartburn/flatulence [40], diarrhea [42], and stomach upset [40, 44]. Three hyperlipidemiafocused RCTs reported their study limitations including small sample size, lack of balanced baseline data between the CHM and control groups and no record of the participants' dietary control [44, 45, 47]. As for risk of bias of the hyperlipidemia-focused RCTs, five trials did not use the random sequence generation method [41-43, 46, 47], only two trials specified the appropriate allocation concealment [44, 45], and six trials failed to employ the blinding of outcome assessment [41-46].

\section{Impaired glucose tolerance}

The seven RCTs on CHM for the treatment of IGT originated from China $(\mathrm{n}=6)$ [48-53] and Australia $(\mathrm{n}=1)$ [54]. Amongst the IGT-focused RCTs, one compared 'CHM' with 'placebo' [54], five compared 'CHM plus lifestyle' interventions with 'lifestyle' interventions alone [48-50, 52, 53], and one compared 'CHM plus lifestyle' intervention with 'placebo plus lifestyle' intervention [51]. The inclusion criteria regarding the $2 \mathrm{hPG}$ level remain stable $(7.8-11.0 \mathrm{mmol} / \mathrm{l})$ while the FPG level is either $<7.0$ or $>7.0 \mathrm{mmol} / \mathrm{l}$ across all the IGT-focused RCTs. Additionally, all the tested CHM products within the IGT-focused RCTs are different. Despite the variation in the inclusion criteria and CHM products, the results on the effects of CHM interventions are consistent throughout all IGT-focused trials. Specifically, more people with IGT reversed to normal in the CHM group (range 19.163.1\%) compared to those in the control group (range 3.1-46.6\%) and less people with IGT progressed to Type 2 diabetes in the CHM group (range 6.2-22.2\%) compared to those in the control group (range 15.3-43.9\%). Of the six IGT-focused RCTs with detailed CHM formulas, five reported the significant between-group effectiveness of the investigated CHM interventions regarding the decrease of FPG, 2hPG, and/or HbA1c levels compared to control interventions [48, 49, 52-54] and Huanglian (黄连) and Gegen (葛根) were the only Chinese herbs both included in these five IGT-focused trials. The sample size of the IGT-focused RCTs ranged from 61 to 514, and all these RCTs provided the age and gender profile of participants in the CHM and control groups (897 males, 939 females, mean age 53 years with the range from 47 to 60 years). The duration of the IGT-focused trials ranged from 3 to 12 months.

All IGT-focused RCTs reported safety-related information and no deaths were noted. The most common side effects reported in the CHM groups were dizziness, gastrointestinal reactions, and abdominal distension. Almost all IGT-related RCTs provided information on their study limitations including a short study period and short follow-up period as well as small sample size. As for risk of bias of the IGT-focused RCTs, three trials provided information about the allocation concealment [51, 52, 54], two trials provided details on the blinding of outcome assessment [51,54], and two trials reported double-blinding of participants and personnel $[51,54]$.

\section{Obesity}

Two RCTs on CHM for the treatment of obesity originated from China [55, 56] and one from Australia [57]. The three obesity-focused trials compared three different CHM products with their placebos. BMI is the key indicator of the inclusion criteria of all obesity-focused RCTs included. However, the threshold value of BMI was set differently across these trials. Amongst the obesityfocused RCTs, CHM products all showed more decrease of body weight than placebos after treatment. Green tea (绿茶) [55, 57] and Juemingzi (决明子) [56, 57] were the Chinese herbs included in two CHM formulas amongst these three obesity-focused trials. The sample size of the obesity-focused RCTs ranged from 78 to 134 and all these RCTs provided the age and gender profile of participants in the $\mathrm{CHM}$ and placebo groups. There were 115 males and 214 females across all the obesity-focused RCTs with a mean age of 40 years, ranging from 39 to 41 years. The duration of the obesity-focused trials ranged from 7 weeks to 6 months.

All obesity-focused RCTs reported safety-related information and no death were noted. CHM interventions were reported more side effects than the placebos, including nausea, headache, and skin rash. One obesityfocused RCT indicated the study limitations including short study period, no follow-up period, and no true placebo group [56]. As for risk of bias of the obesity-focused RCTs, all trials reported the double-blinding of participants and personnel while these trials failed to provide any details of the blinding of outcome assessment.

\section{Combined stroke risk factors}

Six RCTs exploring the efficacy of CHM on one or more of the stroke risk factors were identified in the systematic review. Specifically, one trial examined the ' $\mathrm{CHM}$ 
plus lifestyle' intervention for the treatment of 'IGT and obesity' compared to 'placebo plus lifestyle' intervention, showing significant efficacy on both IGT and obesity before and after treatment and a significant effect on obesity control between groups after treatment [58]; Two trials examined the 'CHM plus biomedicine' interventions for the treatment of 'diabetes and hyperlipidemia' and 'hypertension and hyperlipidemia' compared to the 'biomedicine' intervention [59] and 'placebo plus biomedicine' intervention [60], respectively-both of these studies found similar effect on the combined stroke risk factors between groups after treatment. Moreover, three trials examined the ' $\mathrm{CHM}$, biomedicine plus lifestyle' interventions for the treatment of 'metabolic syndrome' [61], 'hypertension and metabolic syndrome' [62], and 'hypertension and obesity' [63] compared to the 'biomedicine plus lifestyle' interventions with or without placebo, respectively, indicating significant effects on all included stroke risk factors by the CHM interventions compared to the control groups after treatment. Except the Bofutsusho-san (防风通圣散) used in two trials, all the other $\mathrm{CHM}$ interventions involved exploring a combination of multiple stroke risk factors were different and therefore it is unable to report the commonly used Chinese herbs which are vital for the efficacy of combined stroke risk factors across these six RCTs. The sample size of the RCTs focused upon combined stroke risk factors ranged from 20 to 106, and two of these RCTs failed to provide the age and gender profile of participants in the CHM and control groups $[58,60]$. The duration of the RCTs exploring the combined stroke risk factors ranged from 4 to 6 months.

All RCTs focusing upon combined stroke risk factors reported safety-related information and no deaths were noted. Five out of these six RCTs reported that side effects only occurred in the CHM group [58, 60-63] including headache, dizziness, gastrointestinal reactions, and skin allergy. Only two RCTs focusing upon combined stroke risk factors identified their study limitations $[60,63]$, including failure to double-blind the RCT, short study period and carry-over effect. As for risk of bias of the RCTs focusing upon combined stroke risk factors, no trial reported appropriate allocation concealment and blinding of outcome assessment, and two trials were found to have a high risk of bias regarding the random sequence generation $[60,61]$.

\section{Discussion}

This paper reports the first comprehensive systematic review of the literature concerning the use of CHM amongst people at greatest risk(s) of stroke. A number of significant findings from our review are important for future evidence-based planning and priority setting for research in stroke prevention.

Our analyses show some positive efficacy and safety evidence of varied CHM interventions in lowering high blood pressure, high blood glucose, high cholesterol, high body BMI and a combination of multiple stroke risk factors. Importantly, our findings indicate that, compared to biomedicine alone/lifestyle modification alone/biomedicine plus lifestyle intervention, CHM monotherapy may be not sufficient enough for people to obtain their treatment goals when treating hypertension, diabetes, and hyperlipidemia, while an intervention of $\mathrm{CHM}$ as a supplement to biomedicine and/or a lifestyle intervention is more effective in lowering the levels of SBP/DBP, glucose, BMI, TC, 2hPG, and/or HbA1c. These findings from our review are in line with previous systematic reviews on CHM for cardiovascular diseases [12-14]. In addition, the evidence reported in the papers included with regards to the successful reversion from elevated blood glucose level to normal by using CHM interventions suggests that some $\mathrm{CHM}$ products, in combination with a lifestyle intervention, could be considered a potential effective therapeutic regimen for IGT, and these findings are consistent with a Cochrane review on CHM for IGT published in 2009 [13]. Although many RCTs identified in our review demonstrate the therapeutic benefits of $\mathrm{CHM}$ in people with a number of stroke risk factors, there is a lack of replicable evidence on CHM use in combined stroke risk factors. It is worth noting that a CHM product (red yeast rice preparation), a medicinal food [64], has been used several times not only for the management of hypertension but also for hyperlipidemia. However, the control interventions of all RCTs examining the efficacy of this rice preparation are different. Therefore, no trial included in our review paper has tested exactly the same $\mathrm{CHM}$ and control interventions for the treatment of any stroke risk factor(s).

Our findings show a large variation in the sample size and study period across the included RCTs. The potential risks of bias have been reported in the domains of allocation concealment, the blinding of participants and personnel, and/or the blinding of outcome assessment in the included RCTs. Most included trials have reported their safety information. No serious adverse events were noted although some studies showed some moderate side effects in the CHM groups.

Stroke risk factors vary by ethnic groups and such disparities may influence the etiology of stroke and the implementation of stroke prevention programs [65]. Nevertheless, the majority of studies on CHM use for stroke risk factors included in this review were conducted in China on Chinese populations. As such, the results shown in our review paper may not always be directly 
applicable to populations at risk of stroke in other countries beyond China. Furthermore, CHM is often composed of a number of herbs and is prescribed based on the unique Chinese medicine theory-syndrome differentiation. The replicability of these trial designs without Chinese medicine practitioners is therefore difficult.

There are some limitations to our systematic review that should be mentioned. Generalisability of the results from this systematic review is limited. Meanwhile, the overall 'unclear' reporting of research methodology in the included RCTs may limit the quality of the results reported in this review. In addition, our review was restricted to English peer-reviewed journal articles.

\section{Conclusion}

Although the findings in this systematic review with regards to the effect of $\mathrm{CHM}$ for stroke modifiable risk factors should be interpreted with caution, the potential therapeutic benefits of $\mathrm{CHM}$ as a treatment-particularly in combination with biomedicine and/or lifestyle intervention-for different stroke risk factors needs to be further examined by conducting rigorous trials. Future research should be designed and implemented with adequate sample size, detailed reporting of the allocation concealment method, sufficient application of doubleblinding with an adequate placebo and blinding of outcome assessment, and long-term follow-up in different countries. Moreover, it is important for future research on this topic to pay attention to potential drug-herb interactions as a major safety issue in trial design when participants need to take one or more co-administered biomedicine as well as CHM products.

\begin{abstract}
Abbreviations
2hPG: 2-hour postprandial glucose; BMl: body mass index; BP: blood pressure; CHM: Chinese herbal medicine; DBP: diastolic blood pressure; FIN: fasting plasma insulin; FPG: fasting plasma glucose; HbA1c: glycated hemoglobin; HC: hip circumferences; HDL: high-density lipoprotein; HDL-C: high-density lipoprotein cholesterol; HOMA- $\beta$ : homeostatic model assessment $\beta$-cell function; HOMA-IR: homeostatic model assessment insulin resistance; IGT: impaired glucose tolerance; LDL-C: low-density lipoprotein cholesterol; LVMI: left ventricular mass index; MBP: mean blood pressure; OGTT: oral glucose tolerance test; PPG: postprandial plasma glucose; RCT: randomized controlled trial; SBP: systolic blood pressure; TC: total cholesterol; TG: triglyceride; TIA: transient ischaemic attack; TO: original heart rate; WC: waist circumference.
\end{abstract}

\section{Authors' contributions}

DS designed the study. WP, CF and JF conducted the literature search. WP and RL extracted and interpreted the data. WP drafted the manuscript and prepared tables and figures. JA and DS contributed to the critical revisions of the manuscript. All authors read and approved the final manuscript.

\section{Author details}

${ }^{1}$ Australian Research Centre in Complementary and Integrative Medicine (ARCCIM), University of Technology Sydney, Ultimo, NSW, Australia. ${ }^{2}$ Centre for Cardiovascular and Chronic Care, University of Technology Sydney, Ultimo, NSW, Australia. ${ }^{3}$ Australian Research Centre in Complementary and Integrative Medicine (ARCCIM), Faculty of Health, University of Technology Sydney, Level 8, Building 10, 235-253 Jones St, Ultimo, NSW 2007, Australia.
Acknowledgements

Not applicable.

\section{Competing interests}

The authors declare that they have no competing interests.

Availability of data and materials

All data used in this systematic review are fully available in the public domain.

Consent for publication

Not applicable.

Ethics approval and consent to participate

Not applicable.

\section{Funding}

This systematic review was funded by the Nancy and Vic Allen Stroke Prevention Fund.

\section{Publisher's Note}

Springer Nature remains neutral with regard to jurisdictional claims in published maps and institutional affiliations.

Received: 18 July 2017 Accepted: 30 August 2017

Published online: 05 September 2017

\section{References}

1. Kim J, Fann DY, Seet RC, Jo DG, Mattson MP, Arumugam TV. Phytochemicals in ischemic stroke. Neuromol Med. 2016;18:283-305.

2. Mukherjee D, Patil CG. Epidemiology and the global burden of stroke. World Neurosurg. 2011;76:S85-90.

3. Feigin VL, Roth GA, Naghavi M, Parmar P, Krishnamurthi R, Chugh S, et al. Global burden of stroke and risk factors in 188 countries, during 1990-2013: a systematic analysis for the Global Burden of Disease Study 2013. Lancet Neurol. 2016;15:913-24.

4. Holloway RG, Benesch C, Rush SR. Stroke prevention: narrowing the evidence-practice gap. Neurology. 2000;54:1899-906.

5. Straus SE, Majumdar SR, McAlister FA. New evidence for stroke prevention: scientific review. JAMA. 2002;288:1388-95.

6. Collaboration Blood Pressure Lowering Treatment Trialists'. Effects of ACE inhibitors, calcium antagonists, and other blood-pressure-lowering drugs: results of prospectively designed overviews of randomised trials. Lancet. 2000;356:1955-64.

7. Goldstein LB, Adams R, Alberts MJ, Appel LJ, Brass LM, Bushnell CD, et al. Primary prevention of ischemic stroke: a guideline from the American heart association/American stroke association stroke council: cosponsored by the atherosclerotic peripheral vascular disease interdisciplinary working group; cardiovascular nursing council; clinical cardiology council; nutrition, physical activity, and metabolism council; and the quality of care and outcomes research interdisciplinary working group: The American academy of neurology affirms the value of this guideline. Stroke. 2006;37:1583-633.

8. Wang J, Xiong X. Outcome measures of Chinese herbal medicine for hypertension: an overview of systematic reviews. Evid Based Complement Alternat Med. 2012:2012:7.

9. Hu J, Zhang J, Zhao W, Zhang Y, Zhang L, Shang H. Cochrane systematic reviews of Chinese herbal medicines: an overview. PLOS ONE. 2011;6:e28696

10. National Center for Complementary and Integrative Health. Traditional Chinese medicine: in depth. 2013. https://nccih.nih.gov/health/ whatiscam/chinesemed.htm. Accessed Oct 2013.

11. Tachjian A, Maria V, Jahangir A. Use of herbal products and potential interactions in patients with cardiovascular diseases. J Am Coll Cardiol. 2010;55:515-25.

12. Liu JP, Zhang M, Wang WY, Grimsgaard S. Chinese herbal medicines for type 2 diabetes mellitus. Cochrane Database Syst Rev. 2004;3:CD003642.

13. Grant SJ, Bensoussan A, Chang D, Kiat H, Klupp NL, Liu JP, et al. Chinese herbal medicines for people with impaired glucose tolerance or impaired fasting blood glucose. Cochrane Database Syst Rev. 2009;4:CD006690. 
14. Liu ZL, Li GQ, Bensoussan A, Kiat H, Chan K, Liu JP. Chinese herbal medicines for hypertriglyceridaemia. Cochrane Database Syst Rev. 2013:6:CD009560.

15. Tong $X$, Dong $L$, Chen $L$, Zhen Z. Treatment of diabetes using traditional Chinese medicine: past, present and future. Am J Chin Med. 2012;40:877-86

16. Sui Y, Zhao H, Wong V, Brown N, Li X, Kwan A, et al. A systematic review on use of Chinese medicine and acupuncture for treatment of obesity. Obes Rev. 2012;13:409-30.

17. Higgins JP, Altman DG, Gøtzsche PC, Jüni P, Moher D, Oxman AD, et al. The Cochrane Collaboration's tool for assessing risk of bias in randomised trials. BMJ. 2011;343:d5928.

18. Lin Z, Xing Z, Cai C, Tan H, Zhang C. Effects of tianma gouteng decoction on the plasma endothelin of patients with primary hypertension of hyperactivity of the liver yang. Chin J Clin Rehabil. 2004;27:5992-3.

19. Li Y. A clinical study on haunglian fire-purging mixture in treatment of 46 cases of primary hypertension. J Tradit Chin Med. 2005;25:29-33.

20. Ye P,Wu C, Sheng L, Li H. Potential protective effect of long-term therapy with Xuezhikang on left ventricular diastolic function in patients with essential hypertension. J Altern Complement Med. 2009;15:719-25.

21. Zhao Y, Liu Y, Guan Y, Liu N. Effect of Yinian Jiangya Yin on primary hypertension in early stage - a clinical observations on 40 patients. J Tradit Chin Med. 2010;30:171-5.

22. Zhong G, Chen M, Luo Y, Xiang L, Xie Q, Li Y, et al. Effect of Chinese herbal medicine for calming Gan (肝) and suppressing hyperactive yang on arterial elasticity function and circadian rhythm of blood pressure in patients with essential hypertension. Chin J Integr Med. 2011;17:414-20.

23. Yang T, Wei J, Lee M, Chen C, Ueng K. A randomized, double-blind, placebo-controlled study to evaluate the efficacy and tolerability of Fufang Danshen (Salvia miltiorrhiza) as add-on antihypertensive therapy in Taiwanese patients with uncontrolled hypertension. Phytother Res. 2012;26:291-8

24. Tong $X$, Lian F, Zhou Q, Xu L, Ji H, Xu G, et al. A prospective multicenter clinical trial of Chinese herbal formula JZQG (Jiangzhuoqinggan) for hypertension. Am J Chin Med. 2013;41:33-42.

25. Wu C, Zhang J, Zhao Y, Chen J, Liu Y. Chinese herbal medicine bushen qinggan formula for blood pressure variability and endothelial injury in hypertensive patients: a randomized controlled pilot clinical trial. Evid Based Complement Alternat Med. 2014;2014:7.

26. Li H, Liu L, Zhao W, Liu J, Yao M, Han Y, et al. Traditional Chinese versus integrative treatment in elderly patients with isolated systolic hypertension: a multicenter, randomized, double-blind controlled trial. J Integr Med. 2010;8:410-6.

27. Chen SL, Liu XY, Xu WM, Mei WY, Chen XL. Clinical study of Western medicine combined with Chinese medicine based on syndrome differentiation in the patients with polarized hypertension. Chin J Integr Med. 2012;18:746-51.

28. Gong C, Huang SL, Huang JF, Zhang ZF, Luo M, Zhao Y, et al. Effects of combined therapy of Xuezhikang Capsule and Valsartan on hypertensive left ventricular hypertrophy and heart rate turbulence. Chin J Integr Med. 2010:16:114-8.

29. Xu Y, Yan H, Yao MJ, Ma J, Jia JM, Ruan FX, et al. Cardioankle vascular index evaluations revealed that cotreatment of ARB Antihypertension medication with traditional Chinese medicine improved arterial functionality. J Cardiovasc Pharmacol. 2013;61:355-60.

30. Chao M, Zou D, Zhang Y, Chen Y, Wang M, Wu H, et al. Improving insulin resistance with traditional Chinese medicine in type 2 diabetic patients. Endocrine. 2009;36:268-74.

31. Ji L, Tong $X$, Wang $H$, Tian $H$, Zhou $H$, Zhang $L$, et al. Efficacy and safety of traditional chinese medicine for diabetes: a double-blind, randomised, controlled trial. PLOS ONE. 2013;8:e56703.

32. Tong XL, Wu ST, Lian FM, Zhao M, Zhou SP, Chen XY, et al. The safety and effectiveness of TM81, a Chinese herbal medicine, in the treatment of type 2 diabetes: a randomized double-blind placebo-controlled trial. Diabetes Obes Metab. 2013;15:448-54

33. Tu X, Xie C, Wang F, Chen Q, Zuo Z, Zhang Q, et al. Fructus Mume formula in the treatment of type 2 diabetes mellitus: A randomized controlled pilot trial. Evid Based Complement Alternat Med. 2013;2013:8.

34. Wu Q, Fan $\mathrm{H}$. The research for the clinical curative effect through combing traditional Chinese medicine with insulin to cure diabetes. Pak J Pharm Sci. 2014;27:1057-61.
35. Cai H, Liu F, Zuo P, Huang G, Song Z, Wang T, et al. Practical application of antidiabetic efficacy of Lycium barbarum polysaccharide in patients with type 2 diabetes. Med Chem. 2015;11:383-90.

36. Lian F, Tian J, Chen X, Li Z, Piao C, Guo J, et al. The efficacy and safety of Chinese herbal medicine Jinlida as add-on medication in type 2 diabetes patients ineffectively managed by metformin monotherapy: a doubleblind, randomized, placebo-controlled, multicenter trial. PLOS ONE. 2015;10:e0130550.

37. Zhang $X$, Liu Y, Xiong D, Xie C. Insulin combined with Chinese medicine improves glycemic outcome through multiple pathways in patients with type 2 diabetes mellitus. J Diabetes Investig. 2015;6:708-15.

38. Hu Y, Zhou X, Liu P, Wang B, Duan D, Guo D. A comparison study of metformin only therapy and metformin combined with Chinese medicine jianyutangkang therapy in patients with type 2 diabetes: a randomized placebo-controlled double-blind study. Complement Ther Med. 2016:24:13-8.

39. Li M, Huang X, Ye H, Chen Y, Yu J, Yang J, et al. Randomized, doubleblinded, double-dummy, active-controlled, and multiple-dose clinical study comparing the efficacy and safety of Mulberry Twig (Ramulus Mori, Sangzhi) Alkaloid Tablet and Acarbose in individuals with type 2 diabetes mellitus. Evid Based Complement Alternat Med. 2016;2016:8.

40. Wang J, Lu Z, Chi J, Wang W, Su M, Kou W, et al. Multicenter clinical trial of the serum lipid-lowering effects of a Monascus purpureus (red yeast) rice preparation from traditional Chinese medicine. Curr Ther Res. 1997;58:964-78.

41. Yang H, Han L, Sheng T, He Q, Liang J. Effects of replenishing qi, promoting blood circulation and resolving phlegm on vascular endothelial function and blood coagulation system in senile patients with hyperlipemia. J Tradit Chin Med. 2006;26:120-4.

42. Ai J, Zhao L, Lu Y, Cai B, Zhang Y, Yang B. A randomized, multicentre, open-label, parallel-group trial to compare the efficacy and safety profile of daming capsule in patients with hypercholesterolemia. Phytother Res. 2009;23:1039-42.

43. Xu CF, Lin XR, Wang YK. Clinical observation on hyperlipemia treated with antihyperlipidemic decoction. J Tradit Chin Med. 2009;29:121-4.

44. Hu M, Zeng W, Tomlinson B. Evaluation of a Crataegus-based multiherb formula for dyslipidemia: a randomized, double-blind, placebo-controlled clinical trial. Evid Based Complement Alternat Med. 2014;2014:365742.

45. Moriarty PM, Roth EM, Karns A, Ye P, Zhao SP, Liao Y, et al. Effects of Xuezhikang in patients with dyslipidemia: a multicenter, randomized, placebo-controlled study. J Clin Lipidol. 2014;8:568-75.

46. Heber D, Yip I, Ashley JM, Elashoff DA, Elashoff RM, Go VL. Cholesterollowering effects of a proprietary Chinese red-yeast-rice dietary supplement. Am J Clin Nutr. 1999;69:231-6.

47. Lin C, Li T, Lai M. Efficacy and safety of Monascus purpureus Went rice in subjects with hyperlipidemia. Eur J Endocrinol. 2005;153:679-86.

48. Wei Y, Hong YZ, Ye X. Effect of Tang No.1 granule in treating patients with impaired glucose tolerance. Chin J Integr Med. 2008;14:298-302.

49. Gao Y, Zhou H, Zhao H, Feng X, Feng J, Li Y, et al. Clinical research of traditional Chinese medical intervention on impaired glucose tolerance. Am J Chin Med. 2013:41:21-32.

50. Fang Z, Zhao J, Shi G, Shu Y, Ni Y, Wang H, et al. Shenzhu Tiaopi granule combined with lifestyle intervention therapy for impaired glucose tolerance: a randomized controlled trial. Complement Ther Med. 2014;22:842-50.

51. Lian F, Li G, Chen X, Wang X, Piao C, Wang J, et al. Chinese herbal medicine Tianqi reduces progression from impaired glucose tolerance to diabetes: a double-blind, randomized, placebo-controlled, multicenter trial. J Clin Endocrinol Metab. 2014;99:648-55.

52. Huang $Y$, Yang $Q$, Wang $H, X u Y$, Peng $W$, Jiang $Y$. Long-term clinical effect of Tangyiping Granules (糖异平颗粒) on patients with impaired glucose tolerance. Chin J Integr Med. 2016;22:653-9.

53. Shi Y, Liu W, Zhang X, Su W, Chen N, Lu S, et al. Effect of Chinese herbal medicine Jinlida granule in treatment of patients with impaired glucose tolerance. Chin Med J. 2016;129:2281-6.

54. Grant SJ, Chang DH, Liu J, Wong V, Kiat H, Bensoussan A. Chinese herbal medicine for impaired glucose tolerance: a randomized placebo controlled trial. BMC Complement Altern Med. 2013;13:104.

55. Pan L, Li D, Lei M, Zhang L, Zhou L. Preparation-containing node of Lotus Rhizome, green tea and Panax notoginseng for obese adults. Chin J Clin Rehabil. 2005;15:231-3. 
56. Zhou Q, Chang B, Chen X, Zhou S, Zhen Z, Zhang L, et al. Chinese herbal medicine for obesity: a randomized, double-blinded, multicenter, prospective trial. Am J Chin Med. 2014;42:1345-56.

57. Lenon GB, Li KX, Chang Y-H, Yang AW, Da Costa C, Li CG, et al. Efficacy and safety of a Chinese herbal medicine formula (RCM-104) in the management of simple obesity: a randomized, placebo-controlled clinical trial. Evid Based Complement Alternat Med. 2012;2012:435702.

58. Hioki C, Yoshimoto K, Yoshida T. Efficacy of bofu-tsusho-san, an oriental herbal medicine, in obese Japanese women with impaired glucose tolerance. Clin Exp Pharmacol Physiol. 2004;31:614-9.

59. Gao F, Hu XF. Effect of Taizhi'an capsule combined with Simvastatin on hyperlipidemia in diabetic patients. Chin J Integr Med. 2006;12:24-8.

60. Poppel PC, Breedveld P, Abbink EJ, Roelofs H, Heerde W, Smits P, et al. Salvia miltiorrhiza root water-extract (Danshen) has no beneficial effect on cardiovascular risk factors. a randomized double-blind cross-over trial. PLoS ONE. 2015;10:e0128695.

61. Chu SL, Fu H, Yang JX, Liu GX, Dou P, Zhang L, et al. A randomized doubleblind placebo-controlled study of Pu'er tea extract on the regulation of metabolic syndrome. Chin J Integr Med. 2011;17:492-8.
62. Chen Y, Fu DY, He YM, Fu XD, Xu YQ, Liu Y, et al. Effects of Chinese herbal medicine Yiqi Huaju Formula on hypertensive patients with metabolic syndrome: a randomized, placebo-controlled trial. J Integr Med. 2013;11:184-94.

63. Azushima K, Tamura K, Haku S, Wakui H, Kanaoka T, Ohsawa M, et al. Effects of the oriental herbal medicine Bofu-tsusho-san in obesity hypertension: a multicenter, randomized, parallel-group controlled trial (ATH-D-14-01021.R2). Atherosclerosis. 2015;240:297-304.

64. Lee C, Jan M, Yu M, Lin C, Wei J, Shih H. Relationship between adiponectin and leptin, and blood lipids in hyperlipidemia patients treated with red yeast rice. Forsch Komplementmed. 2013;20:197-203.

65. Heuschmann PU, Grieve AP, Toschke AM, Rudd AG, Wolfe CD. Ethnic group disparities in 10-year trends in stroke incidence and vascular risk factors. Stroke. 2008;39:2204-10.

\section{Submit your next manuscript to BioMed Central and we will help you at every step:}

- We accept pre-submission inquiries

- Our selector tool helps you to find the most relevant journal

- We provide round the clock customer support

- Convenient online submission

- Thorough peer review

- Inclusion in PubMed and all major indexing services

- Maximum visibility for your research

Submit your manuscript at www.biomedcentral.com/submit 